\title{
Odor Landscapes in Turbulent Environments
}

\author{
Antonio Celani, ${ }^{1,2}$ Emmanuel Villermaux, ${ }^{3}$ and Massimo Vergassola ${ }^{1,4}$ \\ ${ }^{1}$ Institut Pasteur, Research Unit "Physics of Biological Systems", 75724 Paris Cedex 15, France \\ and CNRS UMR 3525, 28 rue du docteur Roux, 75015 Paris, France \\ ${ }^{2}$ The Abdus Salam International Centre for Theoretical Physics (ICTP), \\ Strada Costiera 11, I-34014 Trieste, Italy \\ ${ }^{3}$ Aix Marseille Université, CNRS, Centrale Marseille, IRPHE UMR 7342, 13384 Marseille, France \\ and Institut Universitaire de France, 75005 Paris, France \\ ${ }^{4}$ Department of Physics, University of California San Diego, La Jolla, California 92093, USA \\ (Received 12 May 2014; revised manuscript received 22 September 2014; published 28 October 2014)
}

The olfactory system of male moths is exquisitely sensitive to pheromones emitted by females and transported in the environment by atmospheric turbulence. Moths respond to minute amounts of pheromones, and their behavior is sensitive to the fine-scale structure of turbulent plumes where pheromone concentration is detectible. The signal of pheromone whiffs is qualitatively known to be intermittent, yet quantitative characterization of its statistical properties is lacking. This challenging fluid dynamics problem is also relevant for entomology, neurobiology, and the technological design of olfactory stimulators aimed at reproducing physiological odor signals in well-controlled laboratory conditions. Here, we develop a Lagrangian approach to the transport of pheromones by turbulent flows and exploit it to predict the statistics of odor detection during olfactory searches. The theory yields explicit probability distributions for the intensity and the duration of pheromone detections, as well as their spacing in time. Predictions are favorably tested by using numerical simulations, laboratory experiments, and field data for the atmospheric surface layer. The resulting signal of odor detections lends itself to implementation with state-of-the-art technologies and quantifies the amount and the type of information that male moths can exploit during olfactory searches.

DOI: 10.1103/PhysRevX.4.041015

\section{INTRODUCTION}

Sex pheromones provide arguably the most striking example of long-range communication through specialized airborne messengers [1]. Most Lepidoptera are consistently attracted to calling females from distances going as far as several hundred meters, reaching their partners in a few minutes [2]. This feat is impressive as females broadcast their pheromone message into a noise-ridden transmission medium (the turbulent atmospheric surface layer), and receiver males face the challenge of extracting information about the female's location from a signal that is attenuated, garbled, and mixed with other olfactory stimuli (see Fig. 1).

The pheromone communication system is under strong evolutionary pressure. This is particularly evident for adult moths of the family Saturniidae and Bombycidae (e.g., the Indian Luna and the silk moth, respectively), which have a lifespan of a few days as adults. Subsisting on stored lipids acquired during the larval stage, they largely devote their

Published by the American Physical Society under the terms of the Creative Commons Attribution 3.0 License. Further distribution of this work must maintain attribution to the author(s) and the published article's title, journal citation, and DOI.
Subject Areas: Biological Physics, Fluid Dynamics

adulthood to the task of reproduction. The result of natural selection is an olfactory system exquisitely sensitive to pheromones: Just a few molecules impinging on the antenna of a male moth are sufficient to alert the insect and trigger a change in its cardiac frequency [5]; concentrations of a few hundred molecules per cubic centimeter elicit specific behavioral responses that prelude flight [6].

The quality and the time course of the pheromone signal matter, in addition to its intensity. As for the quality, the signal is usually a blend of two or more chemical compounds. Species of closely related families often use similar components, and discrimination is achieved by different combinations and/or ratios in the mixture. Pheromone components of sympatric species that emit similar pheromone blends often act as behavioral antagonists [7], and the discrimination among different blends is extremely fine [8]. The first-order center for the discrimination is the macroglomerular complex of the antennal lobe, where detections from olfactory receptor neurons are integrated [9]. As for the time course of the signal, turbulence strongly distorts the pheromone signal, leading to wildly intermittent fluctuations of concentration at large distances from the source. As shown in Fig. 1, the signal features alternating bursts and clean-air periods with a broad spectrum of durations [10]. 


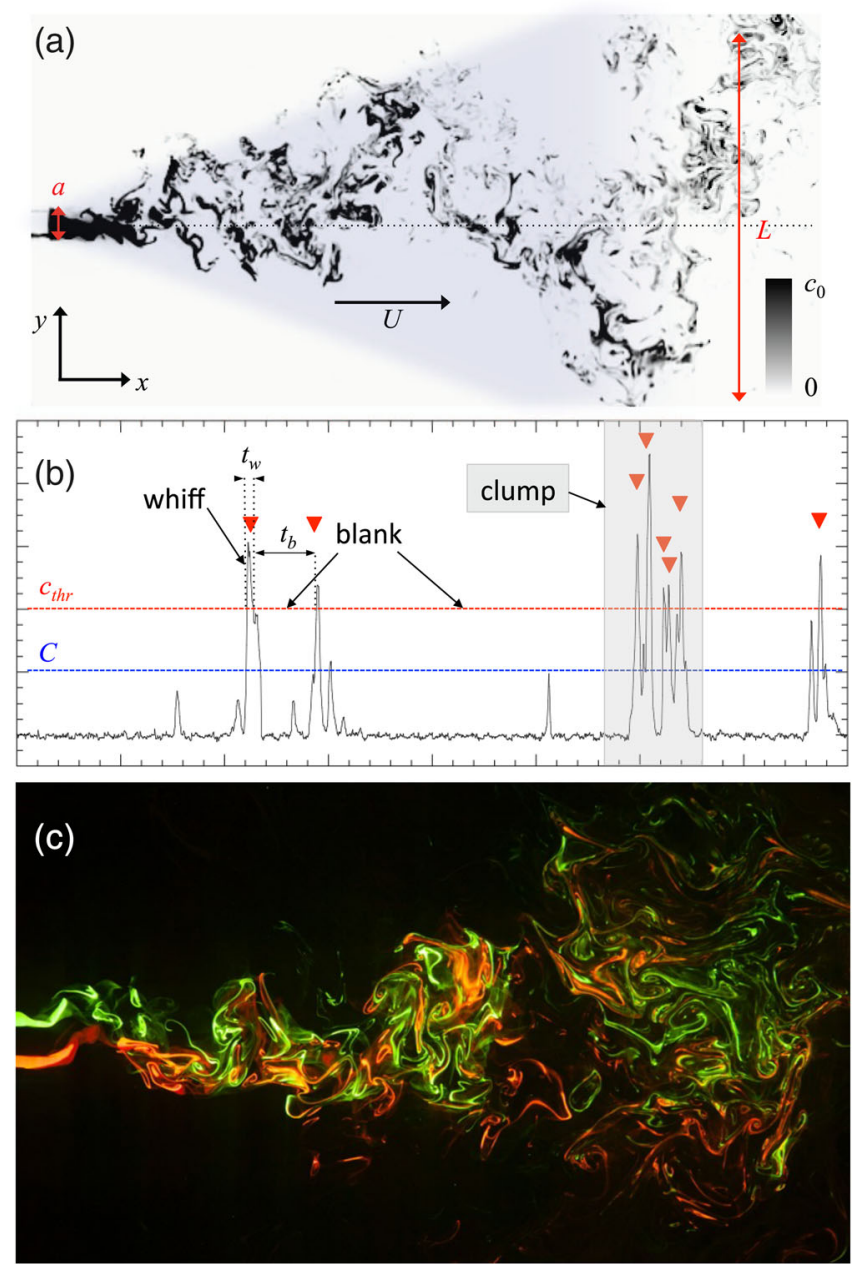

FIG. 1. The structure of a turbulent odor plume. (a) A twodimensional section of a plume from the jet-flow experiment [3]. The shaded area is the projection of the conical average plume, i.e., the region outside of which crosswind transport is weak and the odor concentration decays rapidly. (b) A typical time series of the odor concentration at a given point in space [3]. Red triangles indicate the occurrence of whiffs, i.e., intervals when the local concentration is above the threshold $c_{\text {thr }}$ indicated by the red line. For olfactory searches, the threshold is comparable to the sensitivity of the pheromone receptors of the insects. The blue line indicates the average concentration $C$ in the regions where the signal is above the noise level. (c) A two-dimensional section of two blending plumes from the jet-flow experiment [4]. The two different chemicals mix as they progress downwind, and the resulting signal is a blend.

Characterizing the properties of odor detections in turbulent flows is a challenging and fundamental problem in statistical fluid dynamics. Furthermore, intermittency generated by the physics of turbulent transport is crucial for eliciting the appropriate biological behavior. Insects exposed to steady, uniform stimuli briefly move upwind, arrest their flight toward the source, and begin crosswind casting (the typical response to the loss of olfactory cues). Males temporarily resume upwind flight when the stimulus is increased stepwise, and they set into sustained upwind flight when exposed to repeated pulses [11-13]. Hence, the statistics of turbulence-airborne odor stimuli is literally the message sent by female to male moths; it controls their behavior and defines the information that male moths can exploit for their searches [14-17]. Therefore, the longstanding problem of characterizing the statistics of odor detections during olfactory searches is essential to understand the neurobiological response of insects [18]. Additional motivation for considering the problem stems from laboratory experiments using olfactometers and/or tethering. Experiments in Refs. $[19,20]$ have Drosophilae tethered to a wire and assay their responses (electrophysiologically and/or behaviorally) to simple odor stimuli, such as pulses of fixed duration, that are most likely not representative of those experienced in the wild. To determine the statistics of physiological stimuli and then reproduce it in the laboratory would represent major progress and would significantly impact the design of future experimental assays.

Here, we address and answer the following questions: How intermittent is the distribution of pheromones as a function of the downwind or crosswind distance from the source? What are the statistical distributions for the intensity and the duration of odor-laden whiffs, and the duration of clean-air pockets? What is the dependency on the sensitivity threshold? How does turbulence affect the ratio among different components of a blend from emission to reception? Can emissions from multiple sources, with different blend ratios, reach the receiver without being irremediably mixed? Results are obtained by developing a theoretical Lagrangian approach that predicts the salient properties of a tracer emitted by a localized source and transported by a turbulent flow. We focus on a continuously emitting source, yet methods generalize to periodic emissions. Predictions are successfully tested by numerical simulations, and laboratory and field experimental data. Consequences for the neurobiological responses of insects during olfactory searches and for laboratory protocols of olfactory stimulation are discussed in the Conclusions.

\section{THEORETICAL FRAMEWORK}

Definition of the problem.-We consider the emission by a source of linear size $a$ (at the origin $\boldsymbol{x}=\mathbf{0}$ ) of a chemical substance (or a mixture) at a constant rate of $J$ molecules per unit time. The environment transporting the chemical is a turbulent incompressible flow $\boldsymbol{u}(\boldsymbol{x}, t)=\boldsymbol{U}+\boldsymbol{v}(\boldsymbol{x}, t)$. The mean wind is $\boldsymbol{U}=(U, 0,0)$, while $\boldsymbol{v}$ is the turbulent component. The turbulence level $v / U$, which is the ratio between the amplitudes of the turbulent component $\boldsymbol{v}$ and of the mean flow $\boldsymbol{U}$, is assumed to be small in the rest of the paper. We are interested in the time series of the concentration $c$ at a downwind distance $x$ (much larger than $a$ but still smaller than the correlation length $L$ of the flow) and crosswind distance $y$ from the source (see Fig. 1). 
The concentration $c(\boldsymbol{x}, t)$ of the chemical obeys the advection-diffusion equation

$$
\frac{\partial c(\boldsymbol{x}, t)}{\partial t}+\boldsymbol{u}(\boldsymbol{x}, t) \cdot \nabla c(\boldsymbol{x}, t)=\kappa \nabla^{2} c(\boldsymbol{x}, t)+J h_{a}(\boldsymbol{x}),
$$

where $\kappa$ is the molecular diffusivity. The function $h_{a}(\boldsymbol{x})$ is the spatial distribution of the source of size $a$, e.g., a top hat vanishing outside the source $(|\boldsymbol{x}|>a)$ and normalized to unity $\left(\int h_{a}(\boldsymbol{x}) d \boldsymbol{x}=1\right)$.

Quantities of interest.-We derive below the expression for the following observables of the concentration field $c$ at a given spatial location (see Fig. 1): (i) the intermittency coefficient $\chi$ defined as the fraction of time the concentration is nonzero. The smaller this number, the longer the searching insect is exposed to clean air. (ii) Next is the average concentration $C$ taken over periods of time when the signal is nonzero. The value of $C$ determines the typical intensity of concentration in an odor-laden plume and whether or not that level is detectible by the insect, as discussed below. (iii) Then, we have the full statistics of the signal intensity, that is, the probability distribution $p(c)$ of the concentration. Its expression involves $C$ and $\chi$ as fundamental parameters. (iv) Insects are supposed to detect a signal during those intervals of time when the local concentration exceeds some sensitivity threshold $c_{\text {thr }}$. We call those periods "whiffs," while the complementary periods when $c \leq c_{\mathrm{thr}}$ are dubbed "blanks," or "below threshold." The temporal structure of the signal is thus given by $p\left(t_{w}\right)$, the probability distribution of the duration $t_{w}$ of the whiffs, and by $p\left(t_{b}\right)$, the probability distribution of the duration $t_{b}$ of intervals below threshold, which we obtain below.

The Lagrangian approach.-Lagrangian methods (see Refs. [21-26] for introduction and reviews) focus on fluid-parcel trajectories, and the statistics of the concentration field is reconstructed from the properties of a suitable ensemble of trajectories. Lagrangian approaches are alternatives to the Eulerian description, where the main focus is the concentration field itself (as, e.g., in the fluctuating plume model [10]). The two descriptions are formally equivalent, yet they lend themselves to physical approaches that are quite distinct. The Lagrangian reformulation of (1) is

$$
c(\boldsymbol{x}, t)=J \int_{-\infty}^{t} d t^{\prime} \int d \boldsymbol{x}^{\prime} h_{a}\left(\boldsymbol{x}^{\prime}\right) p_{\boldsymbol{v}}\left(\boldsymbol{x}^{\prime}, t^{\prime} \mid \boldsymbol{x}, t\right),
$$

where $p_{v} d x^{\prime}$ is the probability that a fluid parcel transported by the flow is around $\boldsymbol{x}^{\prime}$ at time $t^{\prime}$, given that it is in $x$ at time $t$. The index of $p_{v}$ is meant to stress that the probability is averaged over the molecular noise statistics, but no average is taken over the fluctuating turbulent flow $\boldsymbol{v}$ (more details can be found in Ref. [26]). Equation (2) states that $c(\boldsymbol{x}, t)$ is determined by tracing back in time the trajectories of parcels that end in $x$ at time $t$. The ensemble of those trajectories forms a puff whose center of mass recedes upwind and whose size $r\left(t^{\prime}\right)$ typically grows as $t^{\prime} \rightarrow-\infty$ (see Fig. 2). Depending on the realizations of $\boldsymbol{v}$, two cases can be distinguished: (i) The distance between the center of mass of the puff and the source never becomes smaller than the size of the puff. These are pockets of clean air, where the concentration $c(\boldsymbol{x}, t)$ vanishes, as follows from Eq. (2). (ii) Otherwise, the concentration $c(\boldsymbol{x}, t)$ is nonvanishing.

It follows from Eq. (2) that the value $c(\boldsymbol{x}, t)$ is proportional to the time of overlap between the puff and the source. The problem thus reduces to characterizing the statistics of the corresponding residence time. The turbulent flow that disperses the puff creates convoluted folds of local structures having some directions extended while others are
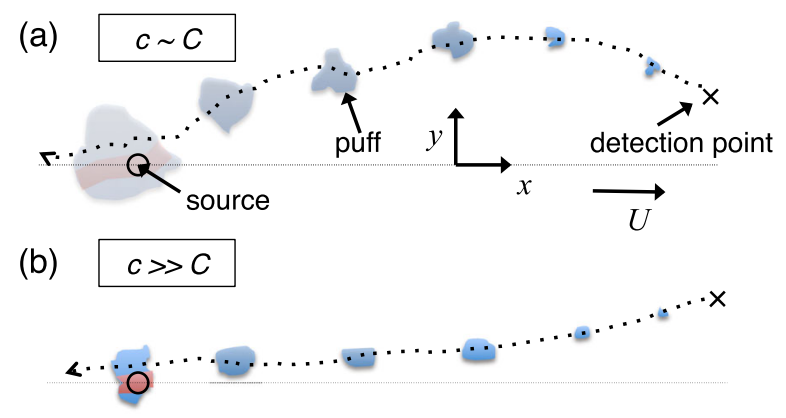

(c)

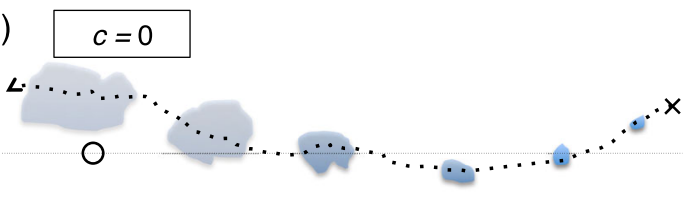

(d)

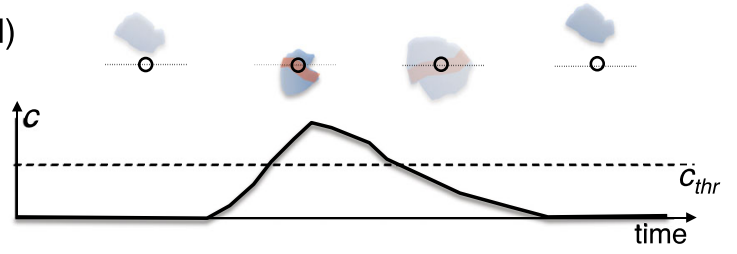

FIG. 2. Scheme of the Lagrangian approach. The concentration $c$ at a given location $\boldsymbol{x}$ and time $t$ is expressed in terms of the history of a Lagrangian puff, that is, an ensemble of particles transported by the turbulent flow, all starting at $\boldsymbol{x}$ at time $t$ and dispersing backwards in time. The concentration $c$ is determined by the size of the Lagrangian puff when it hits the source (if it does): (a) Average values $c \simeq C$ correspond to the puff hitting the source with a typical value of the size; (b) intense concentrations $c$ correspond to the puff hitting the source with unusually small sizes; (c) the concentration $c$ vanishes if the puff never hits the source throughout its history. (d) The sketch of a time series. From left to right: blank, the concentration $c$ vanishes; whiff, the puff hits the source with a small size and $c$ passes the threshold of detection $c_{\text {thr }}$; blank, turbulent diffusion enlarges the size of the puff and $c$ decays below the threshold, then $c$ vanishes because of the puff losing contact with the source. The red strips indicate the regions of the puff overlapping with the source as the puff is swept by the turbulent flow. 
contracted down to the diffusive scale $\eta$ of the scalar concentration field. The specific nature of those structures is determined by the signs of the Lyapunov exponents of the flow. Here, though, we are interested in the statistics of the residence time at the source, the size of which is $a \gg \eta$. Therefore, we physically expect that the small-scale structures of the puff are smoothed out by the integrals appearing in Eq. (2), affecting only constant factors that are not essential for the specific quantities discussed here. In particular, if we disregard constants of order unity, a sufficient characterization of the puffs should be provided by the dynamics of their center of mass and their overall size. We shall derive below the consequences of these physical assumptions and compare the resulting predictions to numerical and experimental data.

Lagrangian properties of the turbulent flow.-We will show shortly that the statistics of odor stimuli for the problem defined above depends on the details of the turbulent flow transporting the pheromones via three exponents: $\alpha, \gamma$, and $\beta$. Power laws are typically observed in turbulent flows as a consequence of scale-invariance properties of fluid dynamical equations [27]. The exponents that we define below are related, respectively, to the dynamics of single-particle, pair dispersion, and rate of growth of the size of a dispersing puff.

(i) The exponent $\alpha$ controls the distance traveled by a single particle at short times $t$ as $(k t)^{1 / \alpha}$, with $k$ constant. The crosswind width of the average plume, outside of which detections are rare, scales with the downwind distance $x$ as $x^{1 / \alpha}$. In most physical cases, the mean wind gives the dominant contribution, so that $\alpha=1, k=U$, and the shape of the average plume is conical. However, for one special case discussed below (the Kraichnan flow), single-particle dispersion is dominated by diffusion at short enough times $(\alpha=2)$, and the standard $U t$ behavior holds only at longer times (yet smaller than those needed to reach the source).

(ii) The exponent $\gamma$ is related to the dispersion of a pair of particles as $\left(k^{\prime} t\right)^{1 / \gamma}$, where $k^{\prime}$ is a constant. For the applications below, the relevant values are $\gamma=2 / 3$, corresponding to the Richardson-Kolmogorov scaling, $\gamma=2$ for ordinary diffusion and $\gamma=1$ for ballistic separation.

(iii) Finally, the exponent $\beta$ is defined by the scaling relation for the rate of growth $\zeta_{r, t} \equiv d \log r / d t=$ $t^{-1}\left(k^{\prime} t / r^{\gamma}\right)^{\beta}$ of a puff of size $r$ at time $t$ after its release. For homogeneous and stationary flow, $\beta=1$ and $\zeta$ depends only on the size. However, if the flow is inhomogeneous, the dependency is more complicated. Namely, in the neutral atmospheric layer, the dynamics explicitly depends on the height; the height of particles released close to the ground grows linearly with time. Nonhomogeneous effects of the height are then conveniently accounted for via the dependency of $\zeta_{r, t}$ on the time $t$ since the release of the puff (we show below that $\beta=2$ in this case). The consistency between the definitions of $\beta$ and $\gamma$ is easy to check: $d r / d t=r \zeta_{r} \sim k^{\prime \beta} t^{\beta-1} r^{1-\beta \gamma}$, and integration of the equation yields $r \sim\left(k^{\prime} t\right)^{1 / \gamma}$ for any $\beta$.

\section{RESULTS: THEORY}

In this section, we summarize the theoretical results about intensity and dynamics of the concentration signal. Derivations are detailed in Appendix A.

The intensity of the concentration signal.-We first consider statistical objects that quantify the concentration $c$ of the pheromones at a given time. The intermittency factor $\chi$ is defined as the fraction of time that $c$ is nonvanishing; the average of the concentration $c$ over that fraction of time is denoted $C$. The threshold of detection, i.e., the minimum concentration that the receiver is able to sense, is denoted $c_{\mathrm{thr}}$. Intervals when $c>c_{\mathrm{thr}}$ are whiffs, while "blanks" or "below threshold" are the complementary regions $c \leq c_{\text {thr }}$ when the signal is either absent or not detectible. The ratio $C / c_{\text {thr }}$ controls whether or not a typical plume is detectible. Using Lagrangian methods, we show in Appendix A that

$$
\begin{aligned}
& \chi=\operatorname{Prob}(c>0) \sim\left(\frac{k^{\prime} x^{1-\gamma}}{U}\right)^{(3-\alpha) / \gamma} f\left(\frac{U y^{\alpha}}{k x}\right), \\
& C=\langle c \mid c>0\rangle \sim \frac{J}{k}\left(\frac{k^{\prime} x}{U}\right)^{-(3-\alpha) / \gamma},
\end{aligned}
$$

where $f$ is a nondimensional function that decays rapidly for large arguments, namely, exponentially in the applications discussed below. Equation (3) indicates that $\chi$ decreases and $C$ remains constant, as $y$ increases. Therefore, moving crosswind away from the mean-wind axis, the signal retains its intensity but becomes sparser. Approaching the source (reducing $x$ ), the intensity within a whiff grows, while the frequency of encounters depends on $\gamma$.

We also show in Appendix A that the concentration $c$ is inversely proportional to the size of the Lagrangian puff (see Fig. 2) when it hits the source. Intense concentrations are associated with flow configurations that leave the puff atypically small. Using the fact that the occurrence of those configurations is a rare event that obeys Poisson statistics, we then show that the tail of the probability distribution $p(c)$ is

$$
p(c) \sim \frac{\chi}{C}\left(\frac{c}{C}\right)^{-2+\frac{\beta \gamma}{3-\alpha}} \exp \left[-\left(\frac{c}{C}\right)^{\frac{\beta \gamma}{3-\alpha}}\right],
$$

for $C \ll c \ll c_{0}$, where $c_{0}$ is the concentration at the source. The moments $\left\langle c^{n}\right\rangle$ are shown [see Eq. (A9)] to depend on $C$ and $\chi$ in Eq. (3) via the relation $\left\langle c^{n}\right\rangle \sim \chi C^{n}$, consistent with the scaling form (4).

The duration of the whiffs.-Since the behavior of insects depends on the time course of the odor stimuli, it is important to characterize the statistics of the whiffs, 
i.e., time intervals when the concentration is above the threshold $c_{\text {thr }}$ of detection. We predict (see Appendix A), for the distribution of the duration $t_{w}$ of the whiffs,

$$
p\left(t_{w}\right) \sim \frac{1}{\tau}\left(\frac{\tau}{t_{w}}\right)^{3 / 2} g_{w}\left(t_{w}\right) .
$$

The power law $-3 / 2$ is cut off by the function $g_{w}$, constant for small arguments, decaying exponentially with a rate $T_{w}^{-1}$ for $t_{w} \gtrsim T_{w}$. The cutoff $T_{w}$ is determined by two physical mechanisms [see Fig. 2(d)]: (i) The flow changes in time, and its new configuration is more effective at dispersing the puff, increasing its size and making the concentration fall below the threshold $c_{\mathrm{thr}}$; (ii) large-scale velocity fluctuations displace the puff away from the source. The expression for the corresponding cutoffs $T_{1}$ and $T_{2}$ is derived in Appendix A, and $T_{w}$ is the minimum between the two. The relative importance of the two mechanisms depends on the details of the flow transporting the odors, on the distance to the source, and on the threshold $c_{\text {thr }}$, as discussed in the examples below.

The power $-3 / 2$ in Eq. (5) originates from the wiggling of the Lagrangian puff in Fig. 2 around the source, leading to the alternation of whiffs (overlaps with the source) and blanks (loss of overlap) distributed according to the properties of a diffusion process. The parameter $\tau$ is the shortest overlap, i.e., the time to diffuse across the size $\simeq a$ of the source. Because of the slow power-law decay $-3 / 2$ in Eq. (5), the average duration is determined by the cutoff: $\left\langle t_{w}\right\rangle \sim T_{w}$.

The duration of intervals below threshold.-The distribution of the duration $t_{b}$ for time intervals when the concentration is below the threshold $c_{\text {thr }}$ is derived in Appendix A as

$$
p\left(t_{b}\right) \sim \frac{1}{\tau}\left(\frac{\tau}{t_{b}}\right)^{3 / 2} g_{b}\left(t_{b}\right) .
$$

Here, $g_{b}$ is approximately constant for durations shorter than the cutoff $T_{b}$, and then it decays exponentially with a rate $T_{b}^{-1}$. The identical $-3 / 2$ power laws in Eqs. (5) and (6) stem from the short-time turbulent diffusion of the Lagrangian puff (see Appendix A for details), which symmetrically loses and gains contact with the source. Note that power laws do not depend on the details of the flow. The temporal structure of whiffs and blanks then contains some information that is independent of environmental variations of the intensity, stratification, and other details of the flow transporting the pheromones.

It follows from Eq. (6) that the average duration of the blanks $\left\langle t_{b}\right\rangle \sim T_{b}$; i.e., it is determined by the cutoff of the distribution, as for the whiffs. Since detections and nondetections are mutually exclusive, their averages (and thus their cutoffs) are not independent, as shown by the exact relation (A16) derived in Appendix A. Specifically, Eqs. (3) and (4) indicate that the value of $C$ and the statistics of $t_{w}$ do not depend on the crosswind distance; i.e., the whiffs do not change in their intensity or duration while moving crosswind. Their frequency does change, though, which is reflected in the intermittency factor $\chi$ in Eq. (3) and affects the statistics of the blanks. In particular, the cutoff $T_{b}$ will grow while moving crosswind according to Eq. (A16).

Clumps of whiffs. - The visual counterpart of the broad distribution (5) for the whiffs is their aggregation in clumps, as in Fig. 1. The short-time diffusion of the Lagrangian puff discussed above (see Appendix A for full details) implies that on/off times within a clump have the same statistics as the time intervals spent above/below zero by a random walk with time step $\tau$. As a result, the total number of whiffs in a clump of size $T_{w}$ is typically $\sqrt{T_{w} / \tau}$, yet their occurrence is highly inhomogeneous. Indeed, it follows from the arcsine law (see, e.g., Ref. [28]) that a time window of extent $\Delta t \ll T_{w}$ centered around a given whiff typically contains $\sqrt{\Delta t / \tau}$ other whiffs. This number is much larger than $\left(\Delta t / T_{w}\right) \sqrt{T_{w} / \tau}$, which would hold for a homogeneous distribution. We conclude that short whiffs tend to cluster and to be interspersed by equally short periods below threshold. Outside of the clusters, large excursions of the Lagrangian puffs generate long whiffs and blanks. Whenever the probability of detecting a whiff is of order unity, $T_{b} \sim T_{w}$, there is symmetry between whiffs and blanks; individual clumps are virtually indiscernible. Conversely, clumps stand out when the detection probability is small-either because the point of detection lies outside of the average plume or because the threshold of detection is large. Clumps are then sparsely distributed as a Poisson process with an expected waiting time between clumps of $\left\langle t_{b}\right\rangle \simeq\left\langle t_{w}\right\rangle / \operatorname{Prob}\left(c>c_{\text {thr }}\right) \gg\left\langle t_{w}\right\rangle$ [see Eq. (A16)], as expected from the Poisson clumping heuristics [29].

Effects of the molecular diffusivity.-Differences in transport among various constituents of a blend are due to their molecular diffusivity $\kappa$. For small volatile compounds, such as pheromones, typical values for $\kappa$ are of the order $10^{-6} \mathrm{~m}^{2} / \mathrm{s}$, corresponding to Péclet numbers $U L / \kappa$ exceeding unity by several orders of magnitude [30]. Values of $\kappa$ do depend on the molecules, though, and their diffusion can thus be different. However, turbulent flows typically lead to the separation of Lagrangian particles (the exponent $\gamma$ is positive). Then, the effects of molecular diffusion are weak for large Péclet numbers, and they are felt only at small separations among particles [26]. The transition between the two regimes of transport occurs at the diffusive scale, which is in the range of a few millimeters to a centimeter (thus, below the size of the source) for relevant flows [30]. We conclude that the statistics of the concentration depends weakly on $\kappa$ and, most importantly, that the species-specific information on the ratios among constituents of a blend of molecules is largely preserved as the mixture is carried by turbulent flow. These conclusions are also supported by experimental 
data on laboratory flows, where the weak dependence on $\kappa$ of the concentration statistics was investigated and quantified [31].

Persistence of odor blends.-When female moths of different species emit blends composed of the same constituents but with different ratios, their messages may interfere and impair the correct decoding by male moths [(see Fig. 1(c)]. The goal of this section is to clarify the conditions ensuring that interference does not occur.

We consider a set of sources of size $a$, spaced by a distance $d \gg a$ from each other, emitting different blends of the same chemical compounds. Each source $k=1,2, \ldots$ releases the chemical species $i=1,2, \ldots$ at a rate $J_{i}^{(k)}$ (all rates are assumed to be comparable). The Lagrangian approach prescribes that we should follow the evolution of a puff released at the detection point and traveling backwards in time. If the puff hits one and only one source, then the resulting signal can be unambiguously attributed to it. Conversely, if the puff traverses two or more sources, the concentration is a mix of their emissions. Given a detection threshold $c_{\text {thr }}$, of the same order for all the components, the probability of receiving a mixed signal equals the probability that a puff crosses two sources while keeping the same (small) size. The condition for a proper identification of the blend is derived in Appendix A and reads

$$
1 \lesssim\left(\frac{R(d)}{r_{\mathrm{thr}}}\right)^{\gamma}=\left(\frac{c_{\mathrm{thr}}}{C(d)}\right)^{\frac{\gamma}{3-\alpha}}=\left(\frac{c_{\mathrm{thr}}}{C(x)}\right)^{\frac{\gamma}{3-\alpha}} \frac{d}{x} .
$$

For typical concentrations, $c_{\mathrm{thr}} \simeq C(x)$, and the probability of receiving a mixed signal reduces to $p_{\text {mix }} \sim$ $\exp \left[-(d / x)^{\beta}\right]$ [see Eq. (A17)]: In order to discriminate two different sources by sampling typical concentrations, their separation $d$ must be comparable to the distance $x$ separating the receiver from one source. Our prediction agrees with experimental observations where the cross correlation between the concentration of two scalars emitted by different sources was measured [4]. Conversely, intense events carry more information and allow us to tell closer sources apart. Indeed, Eq. (7) shows that whiffs with strong concentrations $c \gtrsim c_{0}(d / a)^{-(3-\alpha) / \gamma}$ are unmixed-they carry the proportion of constituents of only one source at any given time. Therefore, the larger the threshold of detection, the greater the power of discrimination (at the expense of sensitivity and time) and vice versa. Even though we have not pursued detailed applications here, Lagrangian methods for the transport of blends can be relevant for the design of mating disruption for pests and diseasetransmitting vectors $[32,33]$.

\section{RESULTS: NUMERICS AND EXPERIMENTS}

To test our predictions, we consider three different types of turbulent flows.
Kraichnan flow.-This is a stochastic velocity field, incompressible, homogeneous and isotropic, with Gaussian statistics, uncorrelated in time, and self-similar Kolmogorov-Richardson spatial scaling (see Ref. [26] for review). These properties correspond to the exponents $\alpha=2, \beta=1$, and $\gamma=2 / 3$ defined in our formulation of the problem. The advantage of this idealized model is that the Lagrangian Monte Carlo method in Ref. [34] allows the numerical simulation of the integer moments of concentration for conditions (namely, the ratio $a / x$ between the size of the source and the distance from it) that are prohibitive for a fully resolved integration of the fluiddynamical equations. In summary, the results for the concentration statistics along the wind axis are (see Appendix B for a detailed derivation)

$$
C(x) \sim x^{-3 / 2} ; \quad \chi \sim \sqrt{\frac{x}{L}} ; \quad\left\langle c^{n}\right\rangle \sim \chi C^{n} \sim x^{-(3 n-1) / 2} .
$$

Figure 3(a) shows that the first four moments are in excellent agreement with the theoretical prediction above.

Jet flow.-This is a laboratory flow qualitatively similar to wind-tunnel experiments. Even though distances from the source are moderate compared to olfactory searches, experimental data still provide a compelling test for our general theory. For the experimental setup in Ref. [3], the single-particle motion is governed by large-scale components of the flow and $\alpha=1$. The main contribution to the dispersion of Lagrangian puffs arises from rapid, smallscale velocity fluctuations that induce a diffusive separation $(\gamma=2)$ with diffusivity $k^{\prime} \sim v a$. Stationarity and homogeneity of the flow ensure $\beta=1$. The function $f$ in Eq. (3) is derived in Appendix B. In addition to a crosswind Gaussian decay, it contains a prefactor $(U / v)^{2}$ which reflects the semiconical shape of the average plume, with aperture angle $v / U$. The area of impact with the source is therefore amplified by $(U / v)^{2}$ with respect to an isotropic distribution. The expressions just listed imply that along the wind axis $y=0$,

$$
C(x) \sim \frac{J}{\operatorname{vax}} ; \quad \chi \sim \frac{U a}{v x} ; \quad\left\langle c^{n}\right\rangle \sim \chi C^{n} \propto x^{-n-1},
$$

with the rate $J \simeq c_{0} U a^{2}$. The scaling of the moments agrees with experimental data in Fig. 3(b). Figure 3(c) presents the distribution of the concentration at various distances along the wind axis, compared to our prediction (4):

$$
p(c) \sim \frac{\chi}{c} \exp \left(-\frac{c}{C}\right)
$$

Experimental data for the duration of whiffs and blanks are compared to Eqs. (5) and (6) in Figs. 3(d), 3(e), and 3(f). 

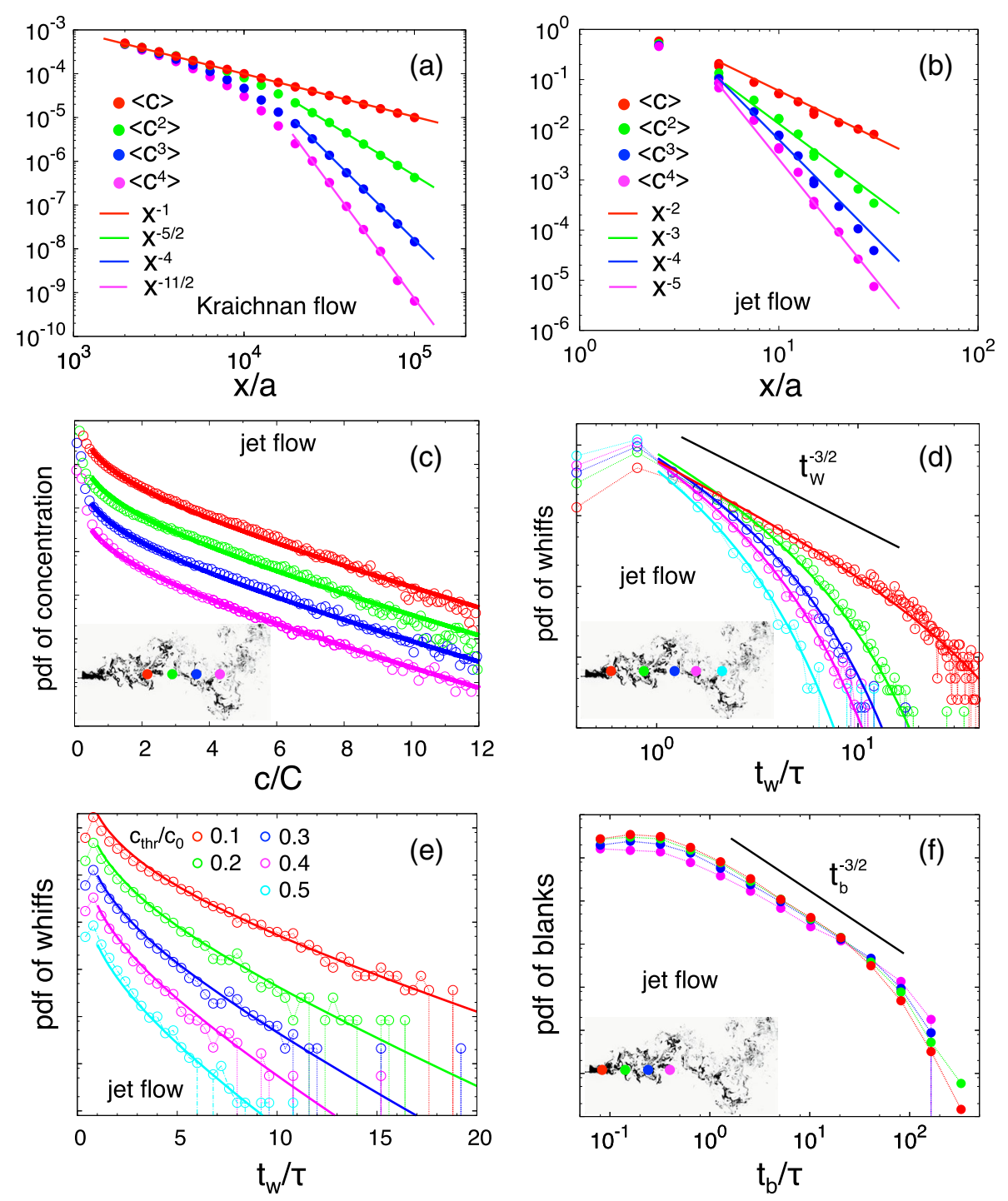

FIG. 3. The statistics of the concentration of odors for the Kraichnan flow [26] [panel (a)] and jet-flow experiments [3] (all other panels). (a) Moments of the concentration for the Kraichnan flow, as a function of the ratio $x / a$ between the distance $x$ to the source and its linear size $a$. Solid lines are the theoretical predictions in Eq. (8). (b) Same as in (a), for the jet-flow experimental data, compared to our predictions (9). (c) The probability density function (pdf) of the concentration (rescaled by its typical value $C$ within the whiffs) at various distances (shown in the inset) from the source, compared to our prediction (10). Data have been shifted vertically for viewing purposes. (d) Pdf for the duration $t_{w}$ of the whiffs (time intervals when the concentration remains above a threshold of detection $c_{\text {thr }}$ ) at various distances from the source. Solid lines are our predictions (5). Durations are rescaled by their most likely value $\tau$. (e) The pdf for the duration of the whiffs vs $c_{\mathrm{thr}}$ (at $x / a=5$ ), compared to our prediction (5). (f) The pdf for the duration of the blanks (intervals without detections) at various distances from the source (shown in the inset), compared to our prediction (6).

The most likely duration $\tau$ is the time to diffuse across the source $\tau \sim a^{2} / k^{\prime} \sim a / v$. We show in Appendix B that the dominant mechanism that cuts off long whiffs is the largescale sweeping of the Lagrangian puffs, and there we provide the expression for the cutoff $T_{w}$ in the exponential function $g_{w}$ in Eq. (5). Blanks obey the power-law predicted by Eq. (6) over nearly two decades. The Poisson clumping regime is realized at distances where detections are sparse and thus $\chi$ is small, i.e., $x \gg a U / v$ along the mean wind axis. In that regime, the exponential in $p(c)$ implies that the average duration of below-threshold intervals depends exponentially on the threshold $c_{\text {thr }} \gtrsim C$ : $\left\langle t_{b}\right\rangle \sim\left\langle t_{w}\right\rangle \exp \left(c_{\text {thr }} / C\right)$. Note that $\left\langle t_{b}\right\rangle$ also grows exponentially with the distance to the source, since $C \propto 1 / x$.

Atmospheric boundary layer.-Finally, we consider the near-neutral atmospheric surface layer [35], the case most directly relevant for olfactory searches. Two particular features of this flow are that (i) the mean wind depends logarithmically on the height $z$ above the ground, and (ii) the intensity $v$ of the velocity fluctuations is nearly constant, yet their correlation length is proportional to $z$. The consequence of (i) is that the time to transport particles 
from the source to the detection point is approximately $t_{\text {hit }} \sim(x / U) \log ^{-1}(z / h)$, where $h$ is the roughness height [35]. The resulting modification to $t_{\text {hit }}$ should a priori be applied to our formulas, but in practice, it is safely ignored as the logarithmic factor varies slowly. Consequences of (ii) are more conspicuous as the increase of the correlation length results in an effective diffusivity $\simeq v z$. Power counting then gives that $z$ is proportional to time, and the growth of the effective diffusivity with $z$ implies the ballistic growth of both the single-particle displacement and the separation between pairs of particles, i.e., $\alpha=\gamma=1$. The rate of growth of a puff of size $r$ is $\zeta_{r} \sim\left(v^{2} t\right) / r^{2}$, which corresponds to $\beta=2$. These scalings are confirmed by experiments with puffs released in the atmospheric surface layer [36].

Inserting the values above into Eq. (3), we obtain (see Appendix B)

$$
\chi \sim \cosh ^{-2}\left(\frac{U y}{v x}\right) ; \quad C \sim c_{0}\left(\frac{U a}{v x}\right)^{2},
$$

i.e., that the intermittency factor $\chi$ is independent of the downwind distance $x$ and decays exponentially in the crosswind direction $y$, as confirmed in Figs. 4(a) and 4(b).
The figures show experimental data $[37,38]$ for the fluctuation intensity $\sigma_{c} /\langle c\rangle \sim \sqrt{\chi^{-1}-1}$, where $\sigma_{c}$ is the standard deviation of the concentration. Equation (3) also predicts, for the typical concentration in a whiff, $C \sim c_{0}(U a / v x)^{2}$, where we estimate, again, $J \simeq c_{0} U a^{2}$. Unfortunately, measurements of absolute concentration are marred by calibration issues [38] so that the prediction cannot be tested directly. However, Eq. (4) predicts, for the tail of the distribution, $p(c) \sim(\chi / c) \exp (-c / C)$, and therefore, the detection probability

$$
\operatorname{Prob}\left(c>c_{\mathrm{thr}}\right) \sim \chi \Gamma\left(0, c_{\mathrm{thr}} / C\right),
$$

where $\Gamma$ is the incomplete Gamma function. The latter quantity is reliably measured, as it depends on ratios of concentration and is in agreement with data shown in Fig. 4(c) from two independent field experiments [37,38].

As for dynamical aspects of the signal, atmospheric data [39] in Fig. 4(d) present a clear power-law distribution of the duration of the whiffs, in agreement with Eq. (5). The typical duration of the whiffs, $\tau \sim a^{2} U /\left(v^{2} x\right)$, is predicted to be independent of the threshold. Comparing the two possible mechanisms for the cutoff of the whiffs (see Appendix B), we find that their average duration is
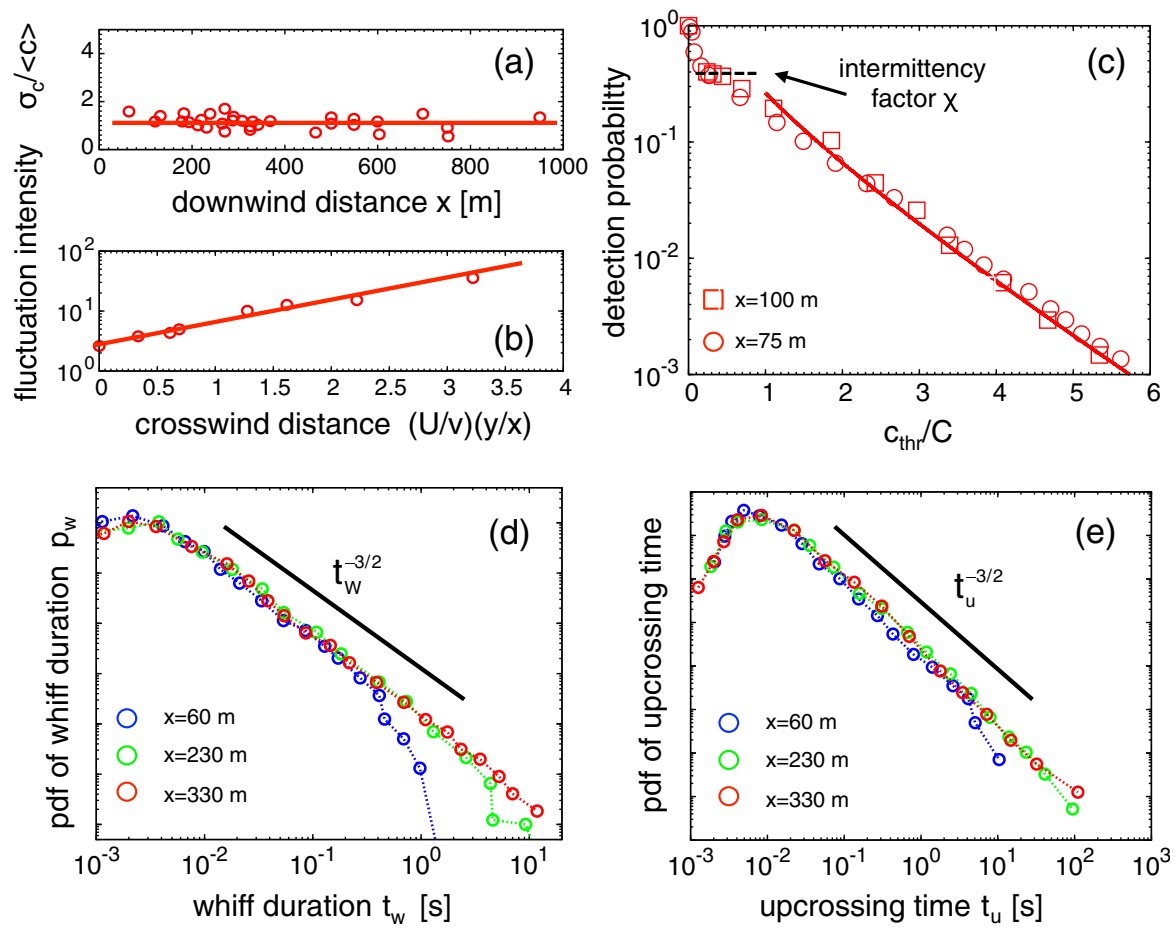

FIG. 4. Odor statistics in the atmospheric surface layer. (a, b) The intensity of the fluctuations of odor concentration as a function of the downwind distance $x$ and of the ratio between the crosswind distance $y$ and $x$, multiplied by the ratio between the mean $U$ and the turbulent component $v$ of the flow transporting the odors. Solid lines are our predictions, from Eq. (11). (c) The probability of detection, i.e., that the local concentration of odors is above a certain threshold, vs the value of the detection threshold. The solid line is the theoretical prediction from Eq. (12). The dashed line is the intermittency factor $\chi=\operatorname{Prob}(c>0)$ in Eq. (11). (d, e) The probability density functions for the duration of the whiffs (time intervals when the concentration remains above the threshold of detection) and the upcrossing time intervals, defined as the time elapsed between the beginnings of two successive whiffs $\left[t_{w}+t_{b}\right.$ in Fig. 1(b)]. The power law $-3 / 2$ is the theoretical prediction derived here. 
determined by the dispersion due to turbulent mixing $\left\langle t_{w}\right\rangle \sim C x / U c_{\mathrm{thr}}$. The cutoff $T_{w}$ for the duration of the whiffs should then linearly increase with the downwind distance. This prediction is in qualitative agreement with experimental data (see Fig. 6 in Ref. [38]); a quantitative comparison would require more statistics, as $T_{w}$ is dominated by low-probability events. Apparently, the statistics of blanks was not measured in field experiments. However, the distribution for the duration of upcrossing intervals $t_{u}$, i.e., the time elapsed between the beginning of two consecutive whiffs, is available from Ref. [39] [see Fig. 4(e)]. Our theory predicts, for $t_{u}$, the same distribution as for the time intervals between odd (or even) zeros of a random walk, which is again a power law $t_{u}^{-3 / 2}$ for $\tau \lesssim t_{u} \lesssim T_{w}$, in agreement with experimental data.

We conclude with a summary of the formulas for the atmospheric boundary layer relevant for the final discussion:

$$
x_{\mathrm{thr}} \simeq \frac{a U}{v} \sqrt{\frac{c_{0}}{c_{\mathrm{thr}}}} ; \quad \tau \simeq \frac{a}{v} \sqrt{\frac{c_{\mathrm{thr}}}{c_{0}}} ; \quad\left\langle t_{w}\right\rangle \simeq \frac{a}{v} \sqrt{\frac{c_{0}}{c_{\mathrm{thr}}}} .
$$

The first equation gives the largest distance $x_{\mathrm{thr}}$ where the conditions $\chi \sim 1$ and $c_{\mathrm{thr}} \simeq C(x)$ are satisfied. The first condition is verified along the wind axis, while the crosswind decay of $\chi$ defines the width of the detection cone $v x / U$. The average duration $\left\langle t_{b}\right\rangle$ of the blanks is comparable to $\left\langle t_{w}\right\rangle$ inside the cone $y / x<v / U$, while $\left\langle t_{b}\right\rangle \gg\left\langle t_{w}\right\rangle$ outside.

\section{DISCUSSIONS AND CONCLUSIONS}

We first consider the implications of our results for the olfactory response of insects. The detection region-where the message sent by female to male moths is least garbled by the turbulence transporting the pheromones-is defined by two conditions: (i) The whiffs of pheromones are sufficiently frequent [that is, the intermittency factor $\chi$ defined in Eq. (3) is not small], and (ii) the typical concentration $C$ in a whiff is detectible; i.e., its ratio $C / c_{\text {thr }}$ with respect to the detection threshold $c_{\text {thr }}$ is not negligible. Experimental measurements show that $B$. mori males respond to air streams containing as little as 200 molecules of bombykol per $\mathrm{cm}^{3}$, corresponding to a sensitivity threshold $c_{\text {thr }} \sim 10^{-18} \mathrm{M}$ [6]. Measured rates for the emission of pheromones by female moths are of the order of a few picograms per second (see, e.g., Ref. [40]), which correspond to an emission rate $J \sim 10 \mathrm{fmol} / \mathrm{s}$ for a molecular weight of a few hundred Daltons, typical for most pheromones. The corresponding concentration at the source is $c_{0} \sim 1 \mathrm{pM}$, for a mean wind $U \sim 1 \mathrm{~m} / \mathrm{s}$ and a size $a$ of the source of a few centimeters, as is typical for female moths.
The physiological parameters above can be inserted into the results for the atmospheric surface layer that we derived here and summarized in Eq. (13). We find that the detection region is a semiconical volume (with aperture angle controlled by the ratio between the intensity of turbulent fluctuations and the mean wind) that extends to downwind distances $x_{\mathrm{thr}} \sim 10^{3} \mathrm{~m}$, in agreement with observations [2]. Hundreds of meters away from the source, the most likely duration $\tau$ of the whiffs is a few milliseconds, which compares well to the shortest pulses detectible by moths [41]. At those distances, whiffs tend to occur in clusters, and a time window of 1 second centered around a detection typically contains $10-20$ odor encounters. This informationridden pattern of stimulation is time integrated at the level of the projection neurons and plays an important role in enhancing the behavioral sensitivity and in promoting exploitative sustained upwind flight $[41,42]$. Upon approaching the source while staying inside the detection cone, the duration of the clumps decreases proportionally to the distance to the source. As a result, the search process is expected to lead to a statistically self-similar set of flight trajectories. Outside the detection cone, periods without any detection of pheromones are typically much longer than the whiffs. Note that even inside the detection cone, periods below threshold might be very long and last up to hundreds of seconds.

Moths switch to exploratory casting when detections become too sporadic (see, e.g., Ref. [43]). While surges are straightforward to define as upwind motion, the trajectories during casting phases are more involved. For example, the angle of flight with respect to the mean wind, the duration of crosswind extensions, and their dependencies on the duration of the ongoing blank period are all factors that potentially affect the patterns of flight during the casting phases. In particular, the extent of the memory of past detections that affect the casting is an open issue. Another open issue is whether or not spatial information on the location of previous detections is involved in the control of the casting (and how, if positive). Search strategies for olfactory robots (see Refs. [44,45]) have shown that extended temporal memory and maps of space do lead to effective searches that alternate surges and casting phases qualitatively resembling those of insects. However, neurobiological constraints were not considered. The upshot is that quantitative data on flight patterns and their relation to the history of detections are needed to make progress on the decision-making processes controlling the casting of insects during their olfactory searches.

Laboratory bioassays with olfactometers and tethered insects are poised to shed light on the previous issues by jointly analyzing the time history of odor stimuli and the virtual flight trajectories of the insects. To ensure that responses observed in the laboratory are informative about the actual behavior of insects, though, it is crucial that stimuli be as close as possible to those experienced by 
insects in the open field. This is the practical level where our results will be useful: Equations (3)-(6) define a complete protocol for generating a sequence of odor pulses. The generation of such stimuli for the neutral atmospheric boundary layer seems achievable mechanically (see Refs. [19,20]) or optogenetically [41], i.e., opening or closing valves that control the delivery of odors or switching on or off light stimulation. For the former, some care should be taken in controlling adsorption effects. It is indeed known that they can lead to delays in the stimulation (see, e.g., Ref. [46]) that could prevent reproducing fast frequencies in the few-ms range that are predicted by Eqs. (5) and (6). No additional stringent limitations seem to prevent reproducing the statistics of Eqs. (3)-(6) in the laboratory. Trains of odor stimuli having such distributions will provide a statistically faithful representation of the landscape of odor detections created by atmospheric turbulence during olfactory searches. The protocol we derived here should then inform the design of future olfactory stimulation assays.

\section{ACKNOWLEDGMENTS}

We thank P. Lucas, J. B. Masson, D. Rochat, and J. P. Rospars for useful discussions. This work was funded by the French State Program Investissements d'Avenir, managed by the Agence Nationale de la Recherche (Grant No. ANR-10-BINF-05).

\section{APPENDIX A: THEORY}

The intensity of the concentration signal.-We first consider the statistics of the pheromone concentration $c$ at a fixed time. If the puff hits the source, it overlaps with it for a time $t_{s}$, which depends on the size $r_{\text {hit }}$ of the puff at the time $t_{\text {hit }}$ of the hit. Since trajectories forming a puff are statistically equivalent and the flow is incompressible, their weight is uniform. Furthermore, for reasons discussed in the main text, we neglect small-scale structures, and we assume that the scaling of the volume of the puff is statistically determined by its size $r_{\text {hit }}$ only; i.e., it is $\propto r_{\text {hit }}^{3}$. Here and in the sequel, we neglect constants of order unity. It follows that the concentration $c$ is the random function

$$
c \propto \begin{cases}J t_{s} r_{\text {hit }}^{-3} & \text { with probability } p_{\text {hit }}, \\ 0 & \text { otherwise }\end{cases}
$$

of the random variable $r_{\text {hit }}$. The expressions for the probability $p_{\text {hit }}$ of hitting the source, $t_{\text {hit }}$ and $t_{s}$, follow from the single-particle dispersion defined in Sec. II. For $r_{\text {hit }} \ll x$, we have

$$
t_{s} \sim \frac{r_{\text {hit }}^{\alpha}}{k} ; \quad t_{\text {hit }} \sim \frac{x}{U} ; \quad p_{\text {hit }} \equiv\left(\frac{r_{\text {hit }}}{x}\right)^{3-\alpha} f\left(\frac{U y^{\alpha}}{k x}\right) .
$$

The expression of $p_{\text {hit }}$ above is justified by the fact that single-particle trajectories dispersing as $t^{1 / \alpha}$ have (fractal) dimension $\alpha$, and the hitting probability then scales with the codimension $3-\alpha$ appearing in $p_{\text {hit }}$ [27]. For $\alpha=2$, $p_{\text {hit }}$ in Eq. (A2) gives the well-known expression for the hitting probability of a random walk with drift, at large distances from the source [47]. The function $f$ in Eq. (A2) is nondimensional and decays rapidly as its argument becomes large, i.e., moving crosswind away from the wind axis.

While the center of the puff is moving backward in time toward the source, its size grows as $r \sim\left(k^{\prime} t\right)^{1 / \gamma}$. The size $r_{\text {hit }}$ when the puff hits the source is expected to have a selfsimilar distribution $[27,48]$; i.e., its expression reads

$$
p\left(r_{\text {hit }}\right)=\frac{1}{R(x)} \phi\left(\frac{r_{\text {hit }}}{R(x)}\right) ; \quad \text { with } \quad R(x) \sim\left(\frac{k^{\prime} x}{U}\right)^{1 / \gamma},
$$

denoting the typical size of the puff at $t_{\text {hit }}$ defined by Eq. (A2). The precise form of $\phi$ is unknown and depends on the details of the turbulent flow, yet its asymptotic behavior is derived as follows. The probability that the size $r_{\text {hit }}$ is well below its typical value $\left[r_{\text {hit }} \leq r \ll R(x)\right]$ is

$$
\int_{0}^{r} p\left(r^{\prime}\right) d r^{\prime} \sim e^{-\int_{0}^{t_{\text {hit }}} \zeta_{r, t^{\prime}} d t^{\prime}} \sim e^{-\left(k^{\prime} t_{\mathrm{hit}}\right)^{\beta} r^{-\beta \gamma}} .
$$

The first step in Eq. (A4) states that the probability that a puff does not grow beyond the size $r$ is given by a Poisson process with local time rates $\zeta\left(t^{\prime}\right)=d \log r\left(t^{\prime}\right) / d t^{\prime}$. The crucial physical ingredient justifying the use of Poisson statistics is that since $r \ll R(x)$, the total time $t_{\text {hit }}$ is much longer than the typical time for growth at the scale $r$. Therefore, the total probability is the product of many largely independent factors. The second step in Eq. (A4) simply follows from the definition of $\beta$. Differentiating Eq. (A4) with respect to $r$ and replacing $t$ by $t_{h i t} \sim x / U$, we finally obtain

$$
\begin{aligned}
p\left(r_{\text {hit }}\right) & \sim\left(\frac{k^{\prime} x}{U r_{\text {hit }}^{\gamma}}\right)^{\beta} \frac{1}{r_{\text {hit }}} \exp \left[-\left(\frac{k^{\prime} x}{U r_{\text {hit }}^{\gamma}}\right)^{\beta}\right] \\
& \Rightarrow \phi(\rho) \sim \rho^{-1-\beta \gamma} \exp \left(-\rho^{-\beta \gamma}\right) \text { for } \rho \ll 1 .
\end{aligned}
$$

Equations (A1) and (A2) imply that the mean concentration $\langle c\rangle=J t_{s} r_{\text {hit }}^{-3} p_{\text {hit }}$ does not depend on $r_{\text {hit }}$, which reflects the conservation of mass. Conversely, averaging $J t_{s} r_{\text {hit }}^{-3}$ with respect to Eq. (A3) [which amounts to replacing $r_{\text {hit }}$ by $R(x)$, apart from numerical factors] gives the conditional average concentration

$$
C(x)=\langle c \mid c>0\rangle \sim \frac{J}{k} R(x)^{\alpha-3} \sim \frac{J}{k}\left(\frac{k^{\prime} x}{U}\right)^{-\frac{3-\alpha}{\gamma}} .
$$


Finally, averaging $p_{\text {hit }}$ in Eq. (A2) over $r_{\text {hit }}$, we obtain, for the intermittency factor $\chi$,

$$
\begin{aligned}
\chi & =\operatorname{Prob}(c>0) \sim\left(\frac{k^{\prime} x^{1-\gamma}}{U}\right)^{\frac{3-\alpha}{\gamma}} f\left(\frac{U y^{\alpha}}{k x}\right) \\
& =\left(\frac{R(x)}{x}\right)^{3-\alpha} f\left(\frac{U y^{\alpha}}{k x}\right),
\end{aligned}
$$

which completes the derivation of Eq. (3).

It follows from Eq. (A1) that the distribution $p(c)$ of the concentration contains two terms. The first is the singular contribution at the origin $\delta(c) \int\left[1-p_{\text {hit }}\right] p\left(r_{\text {hit }}\right) d r_{\text {hit }}$. The second is the continuous contribution $p_{\text {hit }} p\left(r_{\text {hit }}\right)\left|d r_{\text {hit }} / d c\right|$. The relation between $r_{\text {hit }}$ and $c$ is read from the first line in (A1) and more conveniently recast as

$$
r_{\text {hit }}=R(x)\left(\frac{c}{C(x)}\right)^{-\frac{1}{3-\alpha}} .
$$

By using Eqs. (A2), (A3), and (A7), we finally obtain

$$
\begin{aligned}
p(c) & =(1-\chi) \delta(c)+\chi p^{+}(c) \quad \text { with } \\
p^{+}(c) & =\frac{1}{(3-\alpha) C}\left(\frac{c}{C}\right)^{-2-\frac{1}{(3-\alpha)}} \frac{\phi\left(\left(\frac{c}{C}\right)^{-\frac{1}{(3-\alpha)}}\right)}{\int \phi(u) u^{3-\alpha} d u} .
\end{aligned}
$$

Intense concentrations are associated with flow configurations, leaving the puff atypically small [see Eq. (A1)]. Since those rare configurations obey the Poisson asymptotics (A5), the tail of the probability distribution $p(c)$ is Eq. (4).

Finally, it follows from Eq. (A9) that the moments $\left\langle c^{n}\right\rangle$ depend on $C$ and $\chi$ in Eqs. (A6) and (A7) as

$$
\left\langle c^{n}\right\rangle \sim \chi C^{n} \propto x^{-(3-\alpha)(1+(n-1) / \gamma)} .
$$

The duration of the whiffs.-As discussed in the Introduction, the behavior of insects depends on the time course of the odor stimuli. It is therefore important to characterize the statistics of the whiffs, i.e., time intervals when the concentration is above the threshold $c_{\text {thr }}$ of detection. The complementary intervals when $c \leq c_{\text {thr }}$ are dubbed "blanks" or "below threshold." The ratio $C / c_{\text {thr }}$, with $C$ given by Eq. (A6), determines whether a typical plume is detectible. We consider a time $t^{*}$ when the concentration $c(\boldsymbol{x}, t)$ just exceeded the threshold $c_{\mathrm{thr}}$. We are interested in the statistics of the duration $t_{w}$ of the whiff, that is, the time interval such that the concentration stays above the threshold for its whole duration and falls below at $t^{*}+t_{w}$. The singleparticle exponent is taken as $\alpha=1$ since the laboratory and the atmospheric flow that we analyze below have that value.

Let us now derive our prediction (5) for the distribution of the duration of the whiffs. From Eqs. (A1), (A2), and (A8) for $\alpha=1$, a threshold $c_{\text {thr }} \gtrsim C$ is associated with the size of the puff,

$$
r_{\mathrm{thr}} \sim \sqrt{\frac{J}{k c_{\mathrm{thr}}}}=\sqrt{\frac{C(x)}{c_{\mathrm{thr}}}} R(x) .
$$

As time progresses, the turbulent velocity field $v$, as well as the probability $p_{v}$ in Eq. (2), evolve. For two times spaced by $t_{w}$, the two puffs to be tracked are released from the same position but with a delay $t_{w}$, which decorrelates the trajectories of the two puffs as we proceed to quantify. It is convenient to discuss separately the effects of the scales of the turbulent flow that are larger, comparable, or smaller than $r_{t h r}$.

(i) Large-scale (sizes $\gg r_{\text {thr }}$ ) velocity fluctuations transport the puffs almost uniformly, and their major effect is then to displace the puffs. The differential displacement between the puffs released at different times can lead to termination of the whiff by making the later puff (released at $t^{*}+t_{w}$ ) lose overlap with the source [see Fig. 2(d)]. The typical time for the loss of contact is determined by analyzing the dynamics of lateral displacements. The angular size of the puff as seen from the detection point is $r_{\mathrm{thr}} / x$. The trajectories of particles transported by the flow form angles (with respect to the direction of the mean wind) of typical amplitude $v / U$. The angle fluctuates in time with a correlation frequency $v / L$, where $L$ is the correlation length of the flow. The rate of change of the angle is thus $v^{2} / L U$. Finally, we combine the two terms above and insert the expression (A11) of $r_{\text {thr }}$. We conclude that the typical time for a lateral displacement of the puffs leading to a loss of contact with the source is

$$
T_{\text {displace }} \sim \frac{U L}{v^{2}} \frac{r_{\mathrm{thr}}}{x} \simeq \frac{U L}{v^{2}} \frac{R(x)}{x} \sqrt{\frac{C(x)}{c_{\mathrm{thr}}}} .
$$

(ii) Scales comparable to the size of the puff $r_{\text {thr }}$ are less effective at displacing puffs, yet they disperse them, i.e., enlarge their size. That effect can terminate the whiff by making $r_{\text {hit }}>r_{\text {thr }}$ for the later puff released at $t^{*}+t_{w}$ [see Fig. 2(d)]. The characteristic time for the growth of the size of a puff is

$$
T_{\text {disperse }}=\zeta_{r_{\mathrm{tr},}, t_{\mathrm{hit}}}^{-1}=\frac{x}{U}\left(\frac{R(x)}{r_{\mathrm{thr}}}\right)^{-\beta \gamma}=\frac{x}{U}\left(\frac{C(x)}{c_{\mathrm{thr}}}\right)^{\frac{\beta \gamma}{2}},
$$

where we used the definition of $\beta, t_{\text {hit }} \simeq x / U$ and Eq. (A3) for the second equality and Eq. (A11) for the last. For times $t_{w} \gg T_{\text {disperse, }}$ we can treat successive time intervals of length $T_{\text {disperse }}$ as largely independent, again use Poisson statistics [as for Eq. (A4)], and obtain that the probability for the size to remain below $r_{\text {thr }}$ for the whole interval $\left(t^{*}, t^{*}+t_{w}\right)$ is $e^{-\int_{0}^{t_{w}} \zeta_{r_{\text {thr }}, t_{\text {hit }}} d t^{\prime}} \sim e^{-t_{w} / T_{\text {disperse }}}$. A similar reasoning can be used for (i), and it yields an exponential decay as well. 
Both physical mechanisms (i) and (ii) lead to a smaller cutoff as the threshold $c_{\text {thr }}$ is increased. Their relative strength depends on the turbulent flow transporting the odors, on the distance to the source, and on the threshold $c_{\mathrm{thr}}$, as discussed in the examples below. The cutoff $T_{w}$ in Eq. (5) is the minimum between $T_{\text {displace }}$ and $T_{\text {disperse }}$.

(iii) Small scales play a crucial role for the dynamics of two puffs delayed by times $t_{w}<T_{w}$. We are interested in situations where $c_{\mathrm{thr}} \gg C(x)$; i.e., only intense fluctuations are detectible. In the Lagrangian formulation, those rare events correspond to puffs reaching $r_{\text {thr }}$, defined in Eq. (A11), and then maintaining that size for an anomalously long time. Indeed, the typical time to reach the size $r_{\text {thr }}$ is $r_{\text {thr }}^{\gamma} / k^{\prime}$. The time to reach the source is $x / U$. Using Eqs. (A3) and (A11), their ratio is $\left(C(x) / c_{\text {thr }}\right)^{\gamma / 2} \ll 1$; i.e., most of the time to reach the source is spent with sizes $\sim r_{\text {thr }}$.

The characteristic time $\zeta_{r_{\text {thr }}, t} \ll t_{\text {hit }}$ for any time $t<t_{\text {hit }}$. The total displacement of the puff at $t_{\text {hit }}$ is thus the sum of largely uncorrelated events of typical amplitude $r_{\text {thr }}$ and is analogous to a diffusion process with effective diffusivity

$$
D_{\text {thr }} \sim r_{\text {thr }}^{2} \frac{\int_{0}^{t_{\text {hit }}} \zeta_{\text {thr }^{\prime}, t^{\prime}} d t^{\prime}}{t_{\text {hit }}} \sim r_{\text {thr }}^{2} \zeta_{\text {thr },}, t_{\text {hit }} .
$$

We now consider two puffs released with an initial time delay $t_{w}$. The time delay induces an initial spatial separation $\sim U t_{w}$. For the cases we consider, it can be verified that even for the smallest times $\tau$ in Eq. (5), the initial separation $U \tau$ is larger than the viscous scale of the flow. Therefore, velocity fluctuations smaller than the size of the two puffs are uncorrelated since the very beginning of the trajectories. At the time when the size $r_{\text {thr }}$ is reached, the displacement of the two trajectories is $\gtrsim r_{\text {thr }}$, and subsequent displacements are then largely independent. We conclude that at the time $t_{\text {hit }}$ when the puffs reach the source, their centers $\boldsymbol{x}_{c}$ are separated as for a three-dimensional diffusion process with the diffusivity $\sim D_{\text {thr }}$ in Eq. (A14) (we neglect a factor 2, as we have done for all other constants that are of order unity).

The beginning of a whiff occurs when the entire source of size $a$ is barely within the puff released at $t^{*}$; i.e., the center of the source is at a distance $\sim a$ from the boundary of the puff. The end of the whiff occurs when the center of the source first loses overlap with the puff. The centers $\boldsymbol{x}_{c}$ of the puffs released at times later than $t^{*}$ are displaced diffusively with coefficient $D_{\text {thr }}$, as shown above. We conclude that $t_{w}$ is distributed as the first exit time for a diffusing process [28], which obeys the $-3 / 2$ power law in Eq. (5). The shortest time $\tau$ in Eq. (5) corresponds to the fastest exit:

$$
\tau \simeq \frac{a^{2}}{D_{\mathrm{thr}}}=\zeta_{r_{\mathrm{thr}}, t_{\mathrm{hit}}}^{-1}\left(\frac{a}{r_{\mathrm{thr}}}\right)^{2} \propto x^{1-2 / \gamma}\left(\frac{c_{\mathrm{thr}}}{C}\right)^{1-\beta \gamma / 2} .
$$

The time to diffuse across the whole size of the puff is $r_{\mathrm{thr}}^{2} / D_{\mathrm{thr}}=\zeta_{\mathrm{thr}}$, coinciding with the typical time for the dispersion of the puff to larger sizes. For $c_{\text {thr }} \lesssim C$, the size of the puff saturates to its typical value $R(x)$ [see Eq. (A3)], with no dependency on the threshold $c_{\text {thr }}$.

The duration of periods below threshold. - The physical origin of the $-3 / 2$ power law in our prediction (6) is identical to Eq. (5), i.e., the diffusion on rapid time scales of the Lagrangian puff, which symmetrically loses and gains contact with the source. We remark again that the power laws do not depend on features such as intensity, stratification, and other details of the flow transporting the pheromones. It follows from Eq. (6) that the average duration $\left\langle t_{b}\right\rangle \sim T_{b}$; i.e., it is determined by the cutoff of the distribution, as for the whiffs.

Detection and nondetection intervals are mutually exclusive, so their averages (and cutoffs) are not independent. In particular, the probability of detection equals the average fraction of time spent above the threshold $c_{\mathrm{thr}}$ :

$$
\begin{aligned}
\operatorname{Prob}\left(c>c_{\mathrm{thr}}\right) & =\frac{\left\langle t_{w}\right\rangle}{\left\langle t_{w}\right\rangle+\left\langle t_{b}\right\rangle} \Rightarrow T_{b} \sim\left\langle t_{b}\right\rangle \\
& =\left\langle t_{w}\right\rangle \frac{\operatorname{Prob}\left(c \leq c_{\mathrm{thr}}\right)}{\operatorname{Prob}\left(c>c_{\mathrm{thr}}\right)} \sim T_{w} \frac{\operatorname{Prob}\left(c \leq c_{\mathrm{thr}}\right)}{\operatorname{Prob}\left(c>c_{\mathrm{thr}}\right)} .
\end{aligned}
$$

Equation (A6) shows that $C$ [and the statistics of $t_{w}$; see Eq. (5)] does not change with the crosswind distance; i.e., intensity and duration of the whiffs are independent of $y$. Their frequency changes, though, as shown by the intermittency factor $\chi$ in Eq. (A7) and the statistics of the intervals below threshold. Namely, Eq. (A16) indicates that the cutoff $T_{b}$ grows moving crosswind.

Persistence of odor blends.-We consider a set of sources of size $a$, spaced by a distance $d \gg a$ from each other, emitting different blends of the same chemical compounds. Each source $k=1,2, \ldots$ releases the chemical species $i=1,2, \ldots$ at a rate $J_{i}^{(k)}$ (all rates are assumed to be comparable). Equation (2) states that we should follow the evolution of a puff released at the detection point and traveling backwards in time. If the puff hits one and only one source, then the resulting signal can be unambiguously attributed to it. Conversely, if the puff traverses two or more sources, the concentration is a mix of their emissions. Given a detection threshold $c_{\text {thr }}$, of the same order for all the components, the probability of receiving a mixed signal equals the probability that a puff of the size $r_{\text {thr }}$ given by Eq. (A11) crosses two sources while keeping the same size. Clearly, if $r_{\text {thr }} \gtrsim d$, mixing of the signals is almost certain. When $r_{\text {thr }} \lesssim d$, the probability of mixing is the product of the probability that the puff is not dispersed, multiplied by the probability for a particle starting from one source to hit the other. The worst-case scenario is when the various sources are aligned along the mean wind. The probability of a mixed signal is then the product of $p_{\text {hit }}$ in Eqs. (A2) and (A4) (with $x$ replaced by $d, y=0$ and $r=r_{\text {thr }}$ ): 


$$
p_{\text {mix }} \simeq\left(\frac{r_{\mathrm{thr}}}{d}\right)^{3-\alpha} \times \exp \left[-\left(\frac{k^{\prime} d}{U r_{\mathrm{thr}}^{\gamma}}\right)^{\beta}\right]
$$

The mixing probability is small if $r_{\text {thr }} \ll d$ or $r_{\text {thr }} \ll$ $\left(k^{\prime} d / U\right)^{1 / \gamma}$. The right-hand side in the last inequality is recognized by Eq. (A3) as the typical separation $R(d)$ between particles in the time $d / U$ to travel the distance $d$ between the sources. Typically, $R(d) \ll d$ for $\gamma \geq 1$, and the condition for a proper identification of the blend is then Eq. (7), having used the relation (A6) between the size of the puff and concentration.

\section{APPENDIX B: NUMERICS AND EXPERIMENTS}

To test our predictions, we have considered three different types of turbulent flows (Kraichnan flow, jet flow, and neutral atmospheric boundary layer) that we proceed to discuss.

Kraichnan flow.-Kraichnan flow (see Ref. [26] for review) is a stochastic velocity field, incompressible, homogeneous and isotropic, with Gaussian statistics, uncorrelated in time, and self-similar KolmogorovRichardson spatial scaling. The advantage of this idealized model is that the Lagrangian Monte Carlo method in Ref. [34] allows the numerical simulation of the integer moments of concentration for ratios $a / x$ (the size of the source over the distance to it) that are prohibitive for a fully resolved integration of the fluid-dynamical equations.

The (unrealistic) short time correlation of the Kraichnan flow induces the diffusion of single particles [26]. The corresponding diffusivity is $k \sim L^{4 / 3} \sim v L$, where $L$ is the correlation length of the flow and $v$ is the typical amplitude of the Gaussian velocity fluctuations. At short distances, diffusion dominates over the mean wind $U$, which takes over at distances $\sim L v / U$. Parameters are chosen to ensure $x \gg L v / U$ so that the time to reach the source is still $t_{\text {hit }} \simeq x / U$. The single-particle exponents defined in Sec. II are then $\alpha=2$ and $k \sim v L$. The spatial scaling of the velocity differences ensures that pair dispersion obeys the Richardson-Kolmogorov scaling $\gamma=2 / 3$; the constant $k^{\prime} \sim v / L^{1 / 3}$. This scaling behavior holds as long as the separation among the particles remains below $L$ (diffusion sets in at larger separations). Finally, homogeneity and stationarity ensure $\beta=1$. Inserting the values above into Eqs. (A6), (A7), and (A10), we obtain Eq. (8).

The cutoff function $f$ in Eq. (A7) is obtained from results on diffusive processes (see, e.g., Refs. [47] and Supplemental Material [49]) as $f(\xi)=\exp (-\xi / 4)$. The scaling of the moments (8) holds when the typical size of the puffs at $t_{\text {hit }}$ is larger than the size of the source $a$. Otherwise, all the moments $\left\langle c^{n}\right\rangle$ tend to coincide with the probability $p_{\text {hit }}=a / x$ that a diffusing particle hit a sphere of size $a$, starting at a distance $x$ from it. The predictions (8) for the scaling of the first four moments shown in Fig. 3(a) are in excellent agreement with the results of numerical simulations.
The numerical method used to simulate the moments $\left\langle c^{n}\right\rangle$ relies on taking the $n$th power of Eq. (2) and averaging over the velocity field to obtain the moments in terms of Lagrangian trajectories. Using the short correlation of the Kraichnan flow, the trajectories of $n$ particles generated by a Monte Carlo method are sufficient to obtain the $n$ th-order moment [34]. We used a numerical implementation identical to Ref. [34], which we refer to for details.

The new difficulty is that most trajectories miss the source and a small fraction of the statistical realizations contribute. Indeed, Eq. (2) shows that realizations where at least one of the $n$ particles misses the source do not contribute to the moments. We circumvented the problem by using importance sampling [50]. Namely, we chose one reference particle and sampled its trajectories by generating a Brownian bridge (see Ref. [51] and Supplemental Material [49]) between the starting point and the source. This guarantees that the particle hit the source at least once. The time of the first passage at the source is generated from the exact probability distribution, calculated by standard methods (see Supplemental Material [49] for details). The remaining $n-1$ particles evolve according to the exact dynamics for their relative separations, as in Ref. [34].

The general idea of importance sampling [50] is that the quality of a Monte Carlo estimation improves if the auxiliary distribution is more concentrated on the subset of events that substantially contribute to the observable being measured. In our case, the number of statistical samples required for the Monte Carlo estimation of $\left\langle c^{n}\right\rangle$ is reduced by the factor $p_{\text {hit }}$ defined in Eq. (A3). For the simulations in Fig. 3(a), the gain is of the order $x / a \simeq 10^{4}-10^{5}$ along the wind axis, and it further increases with the crosswind distance. Further details on the method can be found in Supplemental Material [49].

Jet flow.-We now consider experimental data for a jet flow [3], a setup qualitatively similar to wind-tunnel experiments. Even though distances from the source are moderate compared to those for olfactory searches by moths, experimental data still provide a compelling test for our general theory. The experimental flow [3] is modeled by the superposition of a mean flow $U \simeq 0.8 \mathrm{~m} / \mathrm{s}$ and a statistically homogeneous, isotropic flow with correlation length $L \simeq$ $8 \mathrm{~cm}$ and intensity of the fluctuations $v / U \simeq 0.25$. The turbulence level is relatively high, which a priori affects our estimates, viz. the time and the probability of hitting the source. Nevertheless, we show below that our predictions agree with experimental data, suggesting that corrections mainly affect constants of order unity, which we disregarded.

Large-scale fluctuations of the flow decorrelate on a time scale $\sim L / v$. At distances $x \lesssim U L / v$, the time to reach the source $t_{\text {hit }} \sim x / U<L / v$, and large-scale diffusion (with diffusivity $\simeq v L$ ), which could potentially dominate the transport of single particles, has not set in yet. For the experiments in Ref. [3], $U L / v$ is about $30 \mathrm{~cm}$, which is 2-3 times bigger than the largest distance to the source 
where measurements are made. It follows that large-scale fluctuations induce a ballistic motion of amplitude $v$ in each realization of the flow (for the relevant times $\lesssim t_{\text {hit }}$ ). Since $v \ll U$, the ballistic contribution by the mean velocity $U$ is stronger. Small-scale fluctuations have shorter correlations and do produce a diffusive motion. However, their diffusivity is $\sim v a$, and the resulting displacement $\sqrt{v a t_{\text {hit }}}$ is negligible compared to $v t_{\text {hit }}$ for $x \gtrsim U a / v$. Since $a$ is a few millimeters, we conclude that single-particle parameters defined in Sec. II are $\alpha=1$ and $k=U$. The main contribution to the dispersion of Lagrangian puffs stems from rapid, small-scale velocity fluctuations that induce a diffusive separation $(\gamma=2)$ with diffusivity $k^{\prime} \sim v a$. The diffusive contribution $\sqrt{v a t_{\text {hit }}}$ dominates Richardson's dispersion $\sqrt{v^{3} t_{\text {hit }}^{3} / L}$ for $x \lesssim(U / v) \sqrt{a L}$. The latter takes over at larger distances. We conclude that for $a \lesssim v x / U \lesssim$ $\sqrt{L a}$, the size of the puff grows diffusively, i.e., $\gamma=2$, $k^{\prime}=v a$. Finally, stationarity and homogeneity of the flow ensure $\beta=1$.

In summary, the parameters defined in Sec. II are $\alpha=1$, $k=U, \gamma=2, k^{\prime}=v a$, and $\beta=1$. Inserting them into Eq. (A6) gives, for the conditional average concentration $C$, the expression (9).

The function $f$ in Eq. (A7) is derived as follows. The probability $p_{\text {hit }}$ of hitting the source is the probability that a spherical puff of size $r_{\text {hit }}$, starting at $(x, y, 0)$ (with $\left.x \gg r_{\text {hit }}\right)$ and moving with constant velocity $\left(-U+v_{x}, v_{y}, v_{z}\right)$, hits the origin. The constancy of the velocity stems from the ballistic motion discussed above. For a given $v$, hitting occurs if at the time $t_{\text {hit }} \simeq x / U$, the distance of the center of the puff from the source is smaller than its radius: $\sqrt{\left(y+v_{y} t_{\text {hit }}\right)^{2}+v_{z}^{2} t_{\text {hit }}^{2}}<r_{\text {hit }}$. The probability of satisfying this inequality in the space $\left(v_{y}, v_{z}\right)$ is calculated for a Gaussian, isotropic distribution of the fluctuations with standard deviation $v \ll U$. Using that the angle formed by the directions of the mean wind and the starting point of the puff is small (since $v / U$ is supposed to be small), we obtain

$$
p_{\mathrm{hit}} \simeq\left(\frac{U r_{\mathrm{hit}}}{v x}\right)^{2} e^{-\left(\frac{U y}{v x}\right)^{2}} ; \quad \chi \sim \frac{U a}{v x} e^{-\left(\frac{U y}{v x}\right)^{2}}
$$

where we omitted constant factors. Along the axis $y=0$, $p_{\text {hit }}$ reduces to the ratio between the cross-sectional area of the puff $\simeq r_{\text {hit }}^{2}$ and the area $\left(v t_{\text {hit }}\right)^{2}$, transverse to the wind axis, spanned by the center of the puff at $t_{\text {hit }}$. Comparing Eq. (B1) to Eq. (A2), we identify the prefactor $(U / v)^{2}$ for the function $f$, which reflects the semiconical shape of the average plume with aperture angle $v / U$. The area of impact with the source is thus amplified by $(U / v)^{2}$ with respect to an isotropic distribution. The second equation in (B1) is obtained using Eq. (A7) and the expression of $f$ just discussed.
The scaling of the moments in Eq. (9) and the prediction (10) are obtained by inserting $C$ from Eq. (9) and $\chi$ from Eq. (B1) into Eqs. (A10) and (4).

As for dynamical aspects of the signal of odors, the two cutoffs discussed in Sec. V read

$$
T_{\text {disperse }} \sim \frac{a c_{0}}{v c_{\text {thr }}} ; \quad T_{\text {displace }} \sim \frac{a U L}{x v^{2}} \sqrt{\frac{c_{0}}{c_{\text {thr }}}} .
$$

The latter is shorter than the former for sufficiently small thresholds, and the cutoff in Eq. (5) is then $T_{w}=T_{\text {displace }}$. The cutoff $T_{b}$ in Eq. (6) for the duration of the blanks follows from the general relation (A16). The shortest duration $\tau$ in Eq. (A15) is $\tau \sim a / v$, independent of the detection threshold and of the distance to the source.

Near-neutral boundary layer.-We finally consider the near-neutral atmospheric surface layer [35]. This is the case most directly relevant for olfactory searches by moths, as they usually search at dusk when convective effects are weak and no stable stratification is present. The latter is more typical at night, while strong convective effects might be present during daytime. Stratification conditions generally depend on micrometeorological conditions such as cloud coverage and humidity. We do not address these aspects here. It is worth noticing that some properties of the odor landscapes, such as the shape of the probability density function of the whiff and blank durations, turn out to be largely insensitive to such details.

Flows in the neutral boundary layer have two special features with respect to the previous cases: (i) The mean wind depends logarithmically on the height $z$ above the ground, viz. $U(z)=(v / \varkappa) \log \left[(z-h) / z_{0}\right]$, where $v$ is the friction velocity, $\varkappa \simeq 0.4$ is the von Karman constant, $z_{0}$ is the roughness height, and $h$ is the displacement height (roughly two-thirds of the canopy height) [35]. Typical values in the atmospheric surface layer are $v \sim 0.1-0.5 \mathrm{~m} / \mathrm{s}$, $z_{0}, h \sim 0.1-1 \mathrm{~m}$ depending on whether the land surface is covered by high grass, pastures, or forests. The consequence of the logarithmic profile is that the time to transport particles from the source to the detection point has a logarithmic dependency on the height. In practice, the resulting modification is safely ignored as the logarithmic factor varies slowly. We shall also omit the order-unity von Karman constant. (ii) The intensity $v$ of velocity fluctuations is nearly constant, yet the size of the largest eddies at height $z$ is $\propto z$ and their correlation time is $z / v$. We are interested in situations where particles are released close to the ground (heights much smaller than the Monin scale of the boundary layer [35]). The variance of the displacement in the height $z$ then behaves as $d z^{2} / d t \sim v z$, i.e., ballistically, because of the effective diffusivity $\simeq v z$. The average height will also systematically increase ballistically. Note that this last statement is due to particles being released close to the ground (the growth of the mean height saturates as its value becomes comparable to the Monin 
scale). Height and time (since the release of the particles) will therefore grow in parallel. Since the effective diffusivity behaves as $v z$, fluctuations in the lateral and longitudinal displacements grow proportionally to $t$ as well. Along the wind direction, the mean wind $U \gg v$ dominates the transport and the hitting time is $t_{\text {hit }} \sim x / U$. Similarly, the sweeping time of a puff of size $r_{\text {hit }}$ across the source is $t_{s} \sim r_{\text {hit }} / U$. The separation between a pair of particles is similarly determined by a height-dependent diffusion process with coefficient $v z$ and therefore scales as $v t$, which gives the exponent $\gamma=1$ and $k^{\prime}=v$. The rate of growth for a puff of size $r$ is $v z / r^{2} \sim v^{2} t / r^{2}$, yielding $\beta=2$.

In summary, the parameters defined in Sec. II are $\alpha=1$, $k=U, \gamma=1, k^{\prime}=v$, and $\beta=2$. These scalings are confirmed by experiments with puffs released in the atmospheric surface layer [36]. It follows from Eq. (A7) and the values above that the typical conditional concentration is given by Eq. (11).

For the intermittency factor $\chi$ in Eq. (A7), we need the form of $f$. The advection-diffusion equation with mean wind $U$ and diffusion coefficient $v z$ is solved in the Supplemental Material [49] by an eigenfunction expansion, as in the simpler case of constant diffusivity. The analytical solution confirms the scalings justified above intuitively and gives

$$
p_{\mathrm{hit}} \simeq\left(\frac{U r_{\mathrm{hit}}}{v x}\right)^{2} \frac{1}{\cosh ^{2}\left(\frac{U y}{v x}\right)} ; \quad \chi \sim \frac{1}{\cosh ^{2}\left(\frac{U y}{v x}\right)} .
$$

The probability $p_{\text {hit }}$ decays exponentially in the crosswind direction $y$, determining the semiconical shape of the average plume, with aperture angle $v / U$. The second equation in (B3) is obtained by using Eq. (A7). The intermittency factor $\chi$ is thus independent of $x$ and decays exponentially in $y$, as confirmed in Figs. 4(a) and 4(b). The data from experiments in Refs. [37,38] report the fluctuation intensity $\sigma_{c} /\langle c\rangle$, where $\sigma_{c}$ is the standard deviation of the concentration. Using Eq. (A10) for the moments of the concentration, $\sigma_{c} /\langle c\rangle \simeq \sqrt{\chi^{-1}-1}$. It follows from Eq. (B3) that in the average plume, $y \lesssim v x / U$, the fluctuation intensity is order unity, while outside the cone, it grows exponentially, as observed in the data. Skewness and kurtosis also grow exponentially with the transverse distance (in agreement with Figs. 2-4 in Ref. [39]).

The tail of the probability density of the concentration follows from Eq. (A9) and the scaling function $\phi(\rho)$ in Eq. (A5). Upon insertion of the appropriate exponents $\alpha=\gamma=1$ and $\beta=2$, we obtain

$$
p(c) \simeq \frac{\chi}{c} e^{-c / C} \quad \text { for } c \gtrsim C,
$$

which yields Eq. (12).
As for dynamical aspects of the signal, from the general expressions (A11), (A3), and (A15), we derive

$$
\zeta_{r, \mathrm{th}_{\mathrm{hit}}} \sim \frac{x}{U}\left(\frac{v}{r}\right)^{2} ; \quad r_{\mathrm{thr}} \simeq \frac{v x}{U} \sqrt{\frac{C(x)}{c_{\mathrm{thr}}}} \simeq a \sqrt{\frac{c_{0}}{c_{\mathrm{thr}}}} ; \quad \tau \sim \frac{a^{2} U}{v^{2} x} .
$$

Comparing the two mechanisms for the cutoff of the whiffs (see Sec. V), we find that

$$
T_{\text {disperse }} \simeq \zeta_{r_{\text {thr }}, t_{\text {hit }}}^{-1} \simeq \frac{r_{\text {thr }}^{2} U}{v^{2} x} ; \quad T_{\text {displace }} \simeq \frac{r_{\mathrm{thr}}}{v} .
$$

For the second equality, we used $U L \simeq v x$ since the integral scale $L$ is proportional to the height $z$, and $z / x \simeq v / U$. The dispersive time is shorter than the displacement time as long as $r_{\text {thr }} \lesssim v x / U$, so that $T_{w} \sim\left\langle t_{w}\right\rangle=$ $T_{\text {disperse. }}$ The cutoff linearly increases with $x$ when the threshold is kept proportional to the typical value $C(x)$ (itself $\propto 1 / x^{2}$ ), with a prefactor inversely proportional to the relative threshold. Our prediction is in qualitative agreement with experimental data (see Fig. 6 in Ref. [38]); a quantitative comparison would require more statistics, as $T_{w}$ is dominated by low-probability events. Apparently, the statistics of periods below threshold was not measured in field experiments. However, the distribution for the duration of upcrossing intervals $t_{u}$, i.e., the time elapsed between the beginning of two consecutive whiffs, is available from Ref. [39]. Our theory predicts for $t_{u}$ the same distribution as for the time intervals between odd (or even) zeros of a random walk, which is again a power law $t_{u}^{-3 / 2}$ for $\tau \lesssim t_{u} \lesssim T_{w}$, in agreement with experimental data in Fig. 4(e). The average duration $T_{w}$ of the blanks follows from Eq. (B6) and the relation (A16).

We conclude with the derivation of the formulas (13) relevant for the final discussion. The first one gives the largest distance $x_{\text {thr }}$ where the two conditions $\chi \sim 1$ and $c_{\mathrm{thr}} \simeq C(x)$ are satisfied. It follows from Eq. (B3) that the first condition is verified along the mean wind axis, while the crosswind decay of $\chi$ defines the width of the detection cone $v x / U$. Equating $C$ in Eq. (11) to $c_{\text {thr }}$, we obtain $x_{\text {thr }}$ in Eq. (13). The second and third equations in (13) are the expressions (B5) of $\tau$ and (B6) of $T_{\text {displace, estimated at }}$ $x=x_{\mathrm{thr}}$. Finally, it follows from Eq. (A16) that the average duration $\left\langle t_{b}\right\rangle$ of the blanks is comparable to $\left\langle t_{w}\right\rangle$ inside the cone $y / x<v / U$, while $\left\langle t_{b}\right\rangle \gg\left\langle t_{w}\right\rangle$ outside.

[1] T. D. Wyatt, Pheromones and Animal Behavior (Cambridge University Press, Cambridge, England, 2003).

[2] C. Wall and J. N. Perry, Range of Action of Moth SexAttractant Sources, Entomologia experimentalis et applicata 44, 5 (1987). 
[3] E. Villermaux and C. Innocenti, On the Geometry of Turbulent Mixing, J. Fluid Mech. 393, 123 (1999).

[4] M. Kree, J. Duplat, and E. Villermaux, The Mixing of Distant Sources, Phys. Fluids 25, 091103 (2013).

[5] A. M. Angioy, A. Desogus, I. Tomassini Barbarossa, P. Anderson, and B.S. Hansson, Extreme Sensitivity in an Olfactory System, Chem. Senses 28, 279 (2003).

[6] J. Boeckh, K. E. Kaissling, and D. Schneider, Insect Olfactory Receptors, Cold Spring Harbor Symp. Quant. Biol. 30, 263 (1965).

[7] T. C. Baker and R. T. Cardé, Analysis of PheromoneMediated Behaviors in Male Grapholitha Molesta, the Oriental Fruit Moth (Lepidoptera: Tortricidae), Environmental entomology 8, 956 (1979).

[8] T. C. Baker, H. J. Fadamiro, and A. A. Cosse, Moth Uses Fine Tuning for Odour Resolution, Nature (London) 393, 530 (1998).

[9] J. A. Riffell, Olfactory Ecology and the Processing of Complex Mixtures, Curr. Opin. Neurobiol. 22, 236 (2012).

[10] J. Murlis, J. S. Elkinton, and R. T. Cardé, Odor Plumes and How Insects Use Them, Annu. Rev. Entomol. 37, 505 (1992).

[11] J. S. Kennedy, A. R. Ludlow, and C. J. Sanders, Guidance of Flying Male Moths by Wind-Borne Sex Pheromone, Physiol. Entomol. 6, 395 (1981).

[12] M. A. Willis and T. C. Baker, Effects of Intermittent and Continuous Pheromone Stimulation on the Flight Behaviour of the Oriental Fruit Moth, Grapholita molesta, Physiol. Entomol. 9, 341 (1984).

[13] T. C. Baker, M. A. Willis, K. F. Haynes, and P. L. Phelan, A Pulsed Cloud of Sex Pheromone Elicits Upwind Flight in Male Moths, Physiol. Entomol. 10, 257 (1985).

[14] W. H. Bossert and E. O. Wilson, The Analysis of Olfactory Communication Among Animals, J. Theor. Biol. 5, 443 (1963).

[15] W. H. Bossert, Temporal Patterning in Olfactory Communication, J. Theor. Biol. 18, 157 (1968).

[16] J. A. Farrell, J. Murlis, X. Long, W. Li, and R. T. Cardé, Filament-Based Atmospheric Dispersion Model to Achieve Short Time-Scale Structure of Odor Plumes, Environ. Fluid Mech. 2, 143 (2002).

[17] W. Li, J. A. Farrell, and R. T. Cardé, Tracking of FluidAdvected Odor Plumes: Strategies Inspired by Insect Orientation to Pheromone, Adaptive Behavior 9, 143 (2001).

[18] J. J. Hopfield, Olfactory Computation and Object Perception, Proc. Natl. Acad. Sci. U.S.A. 88, 6462 (1991).

[19] B. J. Duistermars, D. M. Chow, and M. A. Frye, Flies Require Bilateral Sensory Input to Track Odor Gradients in Flight, Curr. Biol. 19, 1301 (2009).

[20] V. Bhandawat, G. Maimon, M. H. Dickinson, and R. I. Wilson, Olfactory Modulation of Flight in Drosophila Is Sensitive, Selective and Rapid, J. Exp. Biol. 213, 3625 (2010).

[21] P. A. Durbin, A Stochastic Model of Two-Particle Dispersion and Concentration Fluctuations in Homogeneous Turbulence, J. Fluid Mech. 100, 279 (1980).

[22] J. C. R. Hunt, Turbulent Diffusion from Sources in Complex Flows, Annu. Rev. Fluid Mech. 17, 447 (1985).

[23] J. D. Wilson and B. L. Sawford, Review of Lagrangian Stochastic Models for Trajectories in the Turbulent Atmosphere, Bound.-Lay. Meteorol. 78, 191 (1996).
[24] S. B. Pope, Turbulent Flows (Cambridge University Press, Cambridge, England, 2000).

[25] B. I. Shraiman and E. D. Siggia, Scalar Turbulence, Nature (London) 405, 639 (2000).

[26] G. Falkovich, K. Gawędzki, and M. Vergassola, Particles and Fields in Fluid Turbulence, Rev. Mod. Phys. 73, 913 (2001).

[27] U. Frisch, Turbulence (Cambridge University Press, Cambridge, England, 1995).

[28] W. Feller, An Introduction to Probability Theory and Its Applications, Vol. 2 (Wiley, New York, 1971).

[29] D. Aldous, Probability Approximations via the Poisson Clumping Heuristic, Applied Mathematical Sciences, Vol. 77 (Springer-Verlag, New York, 1989).

[30] R. T. Cardé and M. A. Willis, Navigational Strategies Used by Insects to Find Distant, Wind-Borne Sources of Odor, Journal of chemical ecology 34, 854 (2008).

[31] J. Duplat, C. Innocenti, and E. Villermaux, A Nonsequential Turbulent Mixing Process, Phys. Fluids 22, 035104 (2010).

[32] P. Witzgall, L. Stelinski, L. Gut, and D. Thomson, Codling Moth Management and Chemical Ecology, Annu. Rev. Entomol. 53, 503 (2008).

[33] W. Takken and B. G. J. Knols, Strategic Use of Chemical Ecology for Vector-Borne Diseases, in Olfaction in VectorHost Interactions, Ecology and Control of Vector-Borne Diseases, Vol. 2 (Wageningen Academic Publishers, The Netherlands, 2010).

[34] U. Frisch, A. Mazzino, and M. Vergassola, Intermittency in Passive Scalar Advection, Phys. Rev. Lett. 80, 5532 (1998).

[35] J. C. Kaimal and J. J. Finnigan, Atmospheric Boundary Layer Flows: Their Structure and Measurement (Oxford University Press, England, 1994).

[36] E. Yee, P. R. Kosteniuk, and J. F. Bowers, A Study of Concentration Fluctuations in Instantaneous Clouds Dispersing in the Atmospheric Surface Layer for Relative Turbulent Diffusion: Basic Descriptive Statistics, Bound.-Lay. Meteorol. 87, 409 (1998).

[37] K. R. Mylne and P. J. Mason, Concentration Fluctuation Measurements in a Dispersing Plume at a Range up to 1000 m, Q. J. R. Meteorol. Soc. 117, 177 (1991).

[38] E. Yee, P. R. Kosteniuk, G. M. Chandler, C. A. Biltoft, and J. F. Bowers, Statistical Characteristics of Concentration Fluctuations in Dispersion Plumes in the Atmospheric Surface Layer, Bound.-Lay. Meteorol. 65, 69 (1993).

[39] E. Yee, R. Chan, P. R. Kosteniuk, G. M. Chandler, C. A. Biltoft, and J. F. Bowers, Measurements of Level-Crossing Statistics of Concentration Fluctuations in Plumes Dispersing in the Atmospheric Surface Layer, Bound.-Lay. Meteorol. 73, 53 (1995).

[40] T. C. Baker, R. T. Cardé, and J. R. Miller, Oriental Fruit Moth Pheromone Component Emission Rates Measured After Collection by Glass-Surface Adsorption, Journal of chemical ecology 6, 749 (1980).

[41] M. Tabuchi et al., Pheromone Responsiveness Threshold Depends on Temporal Integration by Antennal Lobe Projection Neurons, Proc. Natl. Acad. Sci. U.S.A. 110, 15455 (2013).

[42] A. Mafra-Neto and R. T. Cardé, Fine-Scale Structure of Pheromone Plumes Modulates Upwind Orientation of Flying Moths, Nature (London) 369, 142 (1994). 
[43] T. C. Baker and N. J. Vickers, Pheromone-Mediated Flight in Moths, in Insect Pheromone Research: New Directions, edited by R. T. Cardé and A. K. Minks (Chapman \& Hall, New York, 1997), pp. 232-247.

[44] J. A. Farrell, S. Pang, and W. Li, Chemical Plume Tracing via an Autonomous Underwater Vehicle, IEEE Journal of Oceanic Engineering 30, 428 (2005).

[45] M. Vergassola, E. Villermaux, and B. I. Shraiman, Infotaxis as a Strategy for Searching without Gradients, Nature (London) 445, 406 (2007).

[46] C. Martelli, J. R. Carlson, and T. Emonet, Intensity Invariant Dynamics and Odor-Specific Latencies in Olfactory Receptor Neuron Response, J. Neurosci. 33, 6285 (2013).
[47] L. G. Leal, Advanced Transport Phenomena (Cambridge University Press, Cambridge, England, 2007).

[48] M. Chaves, K. Gawędzki, P. Horvai, A. Kupiainen, and M. Vergassola, Lagrangian Dispersion in Gaussian Self-Similar Velocity Ensembles, J. Stat. Phys. 113, 643 (2003).

[49] See Supplemental Material at http://link.aps.org/ supplemental/10.1103/PhysRevX.4.041015 for further details on the analytical derivations and the numerical method employed for the Kraichnan model.

[50] M. Denny, Introduction to Importance Sampling in Rare Event Simulations, Eur. J. Phys. 22, 403 (2001).

[51] P. Glasserman, Monte Carlo Methods in Financial Engineering (Springer-Verlag, New York, 2004). 